\title{
In situ analysis of intrahepatic virological events in chronic hepatitis B virus infection
}

\author{
Xiaonan Zhang, ${ }^{1}$ Wei Lu, ${ }^{2}$ Ye Zheng, ${ }^{3}$ Weixia Wang, ${ }^{1}$ Lu Bai, ${ }^{1,4}$ Liang Chen, ${ }^{2}$ Yanling Feng, ${ }^{3}$ Zhanqing Zhang, ${ }^{2}$ \\ and Zhenghong Yuan ${ }^{1,4}$
}

${ }^{1}$ Research Unit, Shanghai Public Health Clinical Center, Key Laboratory of Medical Molecular Virology, Shanghai Medical College, Fudan University, Shanghai, China. ${ }^{2}$ Department of Hepatology and ${ }^{3}$ Department of Clinical Pathology, Shanghai Public Health Clinical Center, Fudan University, Shanghai, China. ${ }^{4}$ Key Laboratory of Medical Molecular Virology at the School of Basic Medical Sciences, Shanghai Medical College, Fudan University, Shanghai, China.

\begin{abstract}
Persistent hepatitis B virus (HBV) infection is established by the formation of an intranuclear pool of covalently closed circular DNA (cccDNA) in the liver. Very little is known about the intrahepatic distribution of HBV cccDNA in infected patients, particularly at the single-cell level. Here, we established a highly sensitive and specific ISH assay for the detection of HBV RNA, DNA, and cccDNA. The specificity of our cccDNA probe set was confirmed by its strict intranuclear signal and by a series of Southern blot analyses. Use of our in situ assay in conjunction with IHC or immunofluorescence uncovered a surprisingly mosaic distribution of viral antigens and nucleic acids. Most strikingly, a mutually exclusive pattern was found between HBV surface antigen-positive (HBsA-positive) and HBV DNA- and cccDNA-positive cells. A longitudinal observation of patients over a 1-year period of adeforvir therapy confirmed the persistence of a nuclear reservoir of viral DNA, although cytoplasmic DNA was effectively depleted in these individuals. In conclusion, our method for detecting viral nucleic acids, including cccDNA, with single-cell resolution provides a means for monitoring intrahepatic virological events in chronic HBV infection. More important, our observations unravel the complexity of the HBV life cycle in vivo.
\end{abstract}

\section{Introduction}

The hepatitis B virus (HBV) is a global public health problem, with more than 240 million chronically infected individuals worldwide. Chronic hepatitis B virus (CHB) infection is associated with a high risk of developing complications such as liver fibrosis, cirrhosis, and hepatocellular carcinoma that result in over 780,000 deaths annually (1). HBV is a member of the Hepadnaviridae family of small, hepatotropic DNA viruses that also includes duck hepatitis $\mathrm{B}$ virus (DHBV) and woodchuck hepatitis virus (WHV). HBV has a small $(3.2 \mathrm{~kb})$, partially double-stranded genome and replicates through reverse transcription of pregenomic RNA (pgRNA). The covalently closed circular DNA (cccDNA) in the nucleus, which serves as the template for transcription of pgRNA and other subgenomic RNAs, is initially formed immediately after HBV entry and persists inside the host cells (2). cccDNA is notoriously refractory to the nucleos(t)ide analogs (NAs) commonly used in clinical practice that inhibit the reverse transcriptase activity of HBV polymerase $(3,4)$. Thus, cccDNA has been widely recognized as the molecular basis of $\mathrm{HBV}$ persistence and, as such, also represents a target for next-generation therapeutics.

Despite its central role in the life cycle of HBV and in life-long persistence in patients with $\mathrm{CHB}$, studies of viral or host factors that regulate the abundance and half-life of cccDNA have been hampered by a series of practical difficulties. Although the knowledge gained from the DHBV model provided us with a general

Authorship note: X. Zhang and W. Lu contributed equally to this work.

Conflict of interest: The authors have declared that no conflict of interest exists. Submitted: June 19, 2015; Accepted: December 23, 2015.

Reference information: J Clin Invest. 2016;126(3):1079-1092. doi:10.1172/JCI83339. framework of the formation and maintenance of cccDNA (5), it may not accurately reflect the in vivo situation in $\mathrm{CHB}$ patients due to the significantly divergent viral and host genetic backgrounds. On the other hand, investigations of HBV cccDNA mostly relied on hepatoma cell lines, which differ in many biological aspects from primary hepatocytes and have been shown to be less efficient in cccDNA accumulation $(6,7)$. Other physiologically relevant studies using liver tissues from $\mathrm{CHB}$ patients $(8,9)$ or HBV-infected chimpanzees (10) yielded valuable information but did not fully characterize the manifestation of viral nucleic acids and antigens in the different stages of liver disease. A thorough investigation of the distribution of viral antigens (surface and core antigens, etc.) and nucleic acids (viral RNA, relaxed circular DNA [rcDNA], and, in particular, cccDNA) with single-cell resolution in the livers of $\mathrm{CHB}$ patients would profoundly broaden our understanding of the biology of this obligatory human virus.

Here, we report the establishment of a highly sensitive and specific ISH assay that allowed us to visualize distinct species of HBV DNA, RNA, and cccDNA in liver biopsies from patients with CHB. In particular, through a series of bulk hybridization assays, we confirmed the specificity toward cccDNA of a probe that spans the gap region of the partially double-stranded DNA genome. The Southern blot analyses revealed a fundamental difference in the form and amount of protein-free DNA between infected liver tissue and hepatoma cell lines harboring the HBV genome. On the basis of the ISH assay, we probed the distribution of cccDNA in liver tissues of CHB patients with varied stages of disease progression. The viral RNA and rcDNA were also visualized at the single-cell level. More importantly, when combined with immunodetection of major viral antigens 
(hepatitis B surface antigen [HBsAg] and hepatitis B core antigen $[\mathrm{HBcAg}])$, we observed a highly mosaic distribution pattern of viral nucleic acids and viral antigens. Accumulation of intrahepatic HBV DNA usually coincided with the absence of surface antigen and was not necessarily accompanied by massive cytoplasmic nucleocapsid. The persistence of nuclear cccDNA was confirmed by using serial biopsies taken before and after antiviral therapy.

\section{Results}

Establishment of an ISH method for detection of HBV RNA, DNA, and cccDNA. Considering the heterogeneous nature of liver tissue, which is organized with a fine microanatomy consisting of parenchymal (hepatocytes) and nonparenchymal cells (stellate cells, sinusoidal endothelial cells, Kupffer cells, and other cell types), we sought to probe the intrahepatic distribution of key viral nucleic acids, including cccDNA, with single-cell resolution. To achieve this, we adopted the ViewRNA ISH technology (Panomics, Affymetrix) and modified it to accommodate the visualization of DNA in addition to RNA. Furthermore, due to the strand specificity of the ViewRNA probes, selective hybridization to cccDNA in the nucleus is theoretically possible using a probe set that targets the gap region of the partially double-stranded genome. The general scheme for probe design is illustrated in Figure 1. Three sets of probes that target different regions of the HBV genome were synthesized. Probe set 1 , which is complementary to the plus strand of the HBV sequence (nt 1930-2900, based on GenBank: AB675675), hybridizes to pgRNA and the positive strand of HBV DNA. Probe set 2, which is complementary to the minus strand of the HBV sequence (nt 2957-837), binds solely to HBV DNA. Probe set 3, which targets the gap region of the plus strand in the HBV virion genome (nt 1090-1590), exclusively binds to cccDNA if viral RNAs were eliminated. In addition, this probe set can also be used to visualize the sum of pgRNA and subgenomic RNAs when DNase I pretreatment is used.

We first tested the performance of these probe sets in liver sections from $\mathrm{CHB}$ patients. As shown in Figure 2A, following DNase I pretreatment, hybridization with probe set 1 resulted in strong cytoplasmic colorization, which reflected the pgRNA distribution. When the adjacent sections from the same tissue were pretreated with RNase A before hybridization, we also observed cytoplasmic signal on scattered hepatocytes, indicative of intracellular HBV DNA (Figure 2B). As expected, the combined treatment of DNase I and RNase A removed most of the signal, although a few weak cytoplasmic dots were still present (Figure $2 \mathrm{C}$ ). The DNA specificity of probe set 2 , which was designed to bind to the minus strand of HBV DNA, was confirmed by the elimination of signal by DNase I pretreatment (compare Figure 2, D and E). On the other hand, RNase A (Figure 2F) treatment had no effect on the strong cytoplasmic staining of HBV DNA. To rule out adventitious, nonspecific colorization, we included no probe controls in parallel in adjacent sections from the same tissues. No observable signal was obtained in these samples (data not shown). For probe set 3, when slides were pretreated with DNase I, hybridization resulted in spotty cytoplasmic signals, suggestive of HBV RNAs (Figure 2H). Strikingly, when the sec- tion from the same tissue was pretreated with RNase A, RNase $\mathrm{H}$, and plasmid-safe DNase (PSD) and hybridized with the same probe set, a weak but consistent nuclear colorization signal appeared (Figure 2G). As expected, pretreatment with RNase A and DNase I before hybridization resulted in no colorization (Figure 2I). Curiously, we found that RNase A and PSD cotreatment was insufficient to eliminate cytoplasmic signals, whereas the addition of RNase $\mathrm{H}$ completely removed signals in the cytosol (Supplemental Figure 2; supplemental material available online with this article; doi:10.1172/JCI83339DS1). These results confirmed the existence of RNA-DNA hybrids in HBV-infected hepatocytes (8). In order to rule out nonspecific hybridization of probe set 3 to the host genomic DNA, we performed ISH on sections from HBV-negative human liver tissues and from rat kidney in parallel. No detectable signal was found in either of these tissues (data not shown). In addition, rigorous control hybridizations were also performed, in which unrelated probe sets or non-HBV hepatitis samples were used. These included HBVpositive sections hybridized to the HCV probe (Supplemental Figure 3, A and B) and sections from patients with HCV, autoimmune hepatitis, or drug-induced hepatitis (Supplemental Figure $3, \mathrm{C}-\mathrm{H}$ ) that were hybridized to the HBV DNA or cccDNA probe. None of these experiments yielded visible chromogen deposition. Thus, these results confirmed the high level of specificity of our ISH assays.

It should be noted, however, that our strand-specific probe set 3 was unable to distinguish protein-free rcDNA (PF-rcDNA) $(6,7)$ from cccDNA. PF-rcDNA, which has a near-complete plus strand, existed in hepatoma cell lines harboring the HBV genome (e.g., HepAD38) and in liver tissues infected with HBV. In cell lines, the ratio of PF-rcDNA to cccDNA is 13:1-25:1 (7), while in clinical liver specimens, it is often less than 1 (9). Hence, we continued to test our probe sets in the HepAD38 cells. As expected, hybridization of probe set 2 resulted in strong cytoplasmic signals in HepAD38 cells without doxycycline treatment (Supplemental Figure $4 \mathrm{~A}$ ). In contrast, a very faint signal (mainly in the nucleus) was observed in HepAD38 cells maintained in doxycycline medium (Supplemental Figure 4B). When HepAD38 cells cultured in doxycycline-free medium were hybridized to probe set 3 after RNase A, RNase H, and PSD treatment, we observed spotty signals mainly in the nucleus. However, we also observed cytoplasmic colorization (Supplemental Figure 4D). Similarly, a very weak signal was found in doxycycline-treated HepAD38 cells (Supplemental Figure 4E). As expected, virtually no signal was observed when HepG2 cells were hybridized in parallel (Supplemental Figure 4, C and F).

To further elucidate the quantity of different forms of deproteinated DNA in the livers of CHB patients, we performed modified Hirt's extraction and Southern blot analyses on HepAD38 cells and biopsies from CHB patients. As shown in Figure 3A, we detected the supercoiled form of the protein-free DNA from the biopsies, while the relaxed form was present at a much lower level (lane 2). Its supercoiled nature was further confirmed by its resistance to $\mathrm{T} 5$ exonuclease (lane 3), which digests all forms of DNA except cccDNA, and its linearization with a restriction endonuclease, SpeI (lane 4). By comparison, protein-free DNA from HepAD38 cells mainly existed in the form of PF-rcDNA (lane 


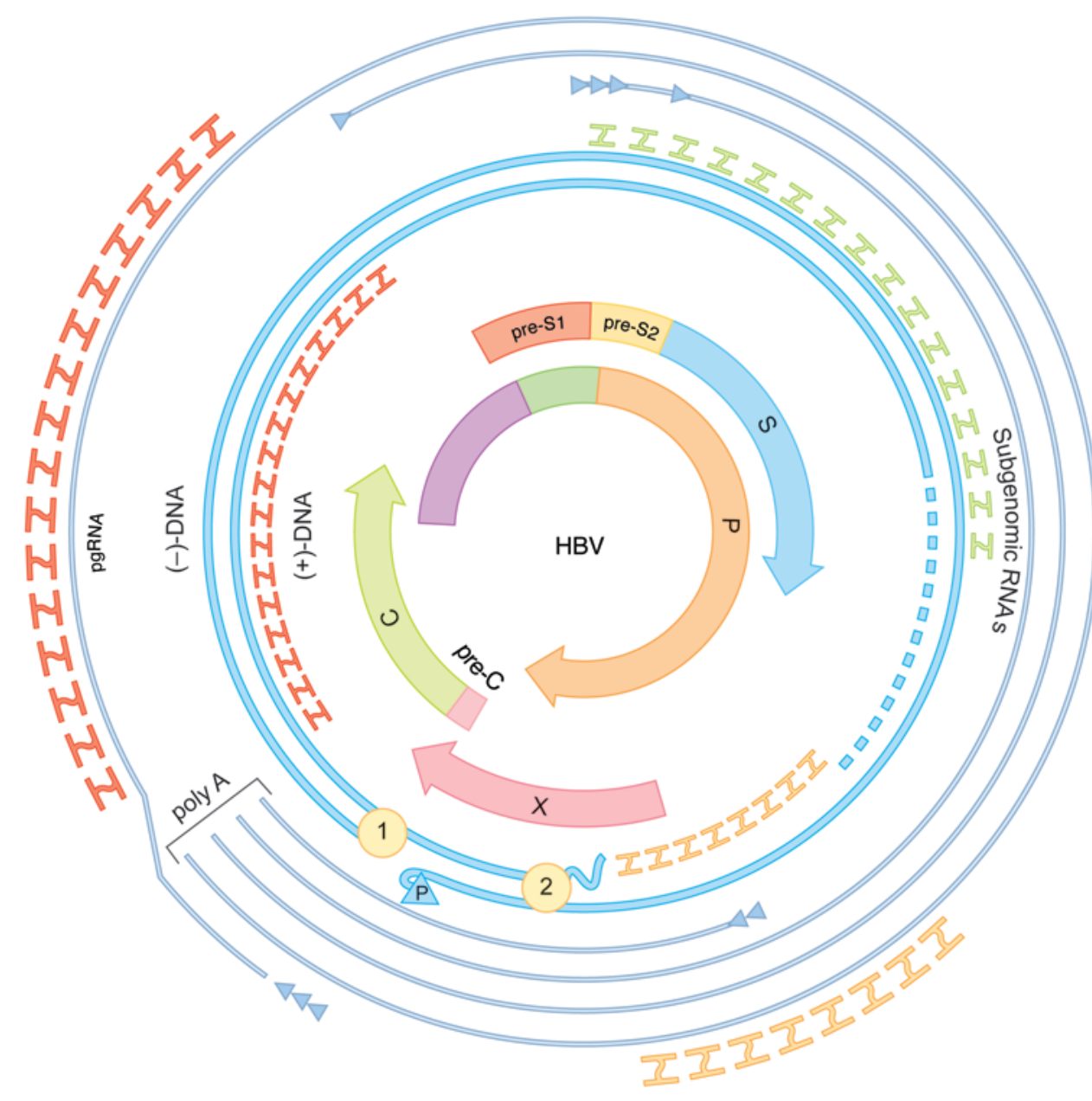

$\llbracket$ Probe set $1 \rrbracket$ Probe set $2 \coprod$ Probe set 3

Figure 1. Schematic illustration of probe set design for the detection of HBV rcDNA, RNA and cccDNA. (+)-DNA, sense strand; (-)-DNA, antisense strand; Poly A, polyadenylated tail; C, HBcAg, P, HBV polymerase; S, HBsAg; X, HBV X protein.

form detected by the minus strandspecific probe (lane 2) was virtually absent when the gap region probe was used (lane 11). Instead, we observed 2 major bands, which were approximately $2.0 \mathrm{~kb}$ and 3.2 $\mathrm{kb}$ (lane 11). PSD treatment did not eliminate these 2 bands but diminished other cross-reactive species (lane 12). Furthermore, T5 exonuclease digestion only left 1 weak band of approximately $2.0 \mathrm{~kb}$ (lane 13), which reconfirmed its cccDNA nature. In HepAD38 cells, 1 major band of rcDNA was detected when the gap region probe was used (lane 14), and PSD treatment slightly reduced other cross-reactive species (lane 15), while T5 exonuclease erased all the signal (lane 16). Moreover, when core particle DNA from liver biopsies and HepAD38 cells was hybridized with these 2 riboprobes (Figure 3D, lanes 8 and 9 and 17 and 18), an ultra-low level of cross-reactivity with the gap region probe was observed in clinical specimens (lane 17), but not in HepAD38 cells (lane 18). This result was also

6), whereas cccDNA was undetectable, presumably due to the inadequate sensitivity of our nonradioactive assay. The relaxed form was degraded by T5 exonuclease and could be linearized by SpeI (lane 8). It should be noted that double-stranded linear DNA (dslDNA) was also present, since SpeI digestion resulted in a 1.2-kb band in addition to 3.2-kb species (lane 8). A heat resistance assay also confirmed the cccDNA form in protein-free DNA from liver biopsies when samples were heated from $75^{\circ} \mathrm{C}$ to $95^{\circ} \mathrm{C}$ (Figure 3B, lanes 2-5), while PF-rcDNA from HepAD38 cells (lanes 6-9) and core particle DNA from a liver biopsy (lanes 11-14) were readily denatured. In addition, topoisomerase I treatment transformed the cccDNA into the relaxed form, as shown in Figure 3C (lanes 1 and 2), while PF-rcDNA from HepAD38 cells showed no mobility change (lanes 4 and 5).

Next, we attempted to further validate the selectivity of our gap-region probe in a bulk hybridization assay. We generated a gap region-specific riboprobe targeting the sequence similar to that of probe set 3. When the total DNA from a biopsy sample and HepAD38 cells was hybridized with minus strand-specific or gap region riboprobe, we observed distinct patterns (Figure 3D, compare lanes 2 and 11). Notably, the intense single-stranded confirmed by additional liver biopsies (Supplemental Figure 5). Interestingly, all this core particle DNA from biopsies did not contain a visible signal above $3.2 \mathrm{~kb}$ ( $\mathrm{rcDNA}$ ), while an intense band of rcDNA was observed in HepAD38 cells. Hence, the band above $3.2 \mathrm{~kb}$, as observed in lanes 11 and 12 (Figure 3D), should be the relaxed form of protein-free DNA, as opposed to cytoplasmic rcDNA, whereas the band in HepAD38 cells (lanes 14 and 15, Figure 3D) should be the sum of PF-rcDNA and cytoplasmic rcDNA.

Collectively, these results indicated that our selective probe sets were capable of visualizing HBV RNA, DNA, and nuclear cccDNA in clinical liver specimens. Southern blot analyses suggested that cccDNA dominated the protein-free DNA and existed at a much higher level in liver biopsies than in HepAD38 cells, which was in line with a previous report (9). Our probe set 3 had an ultra-low level of cross-reactivity with cytoplasmic replicative intermediates in clinical specimens, while the same did not hold true for HepAD38 cells. This observation may explain why the signal generated by probe set 3 was not confined to the nucleus in HepAD38 cells. In liver specimens, the probe set 3 mainly bound to the supercoiled and relaxed forms of protein-free DNA, both of which constitute the nuclear reservoir of viral replication. 


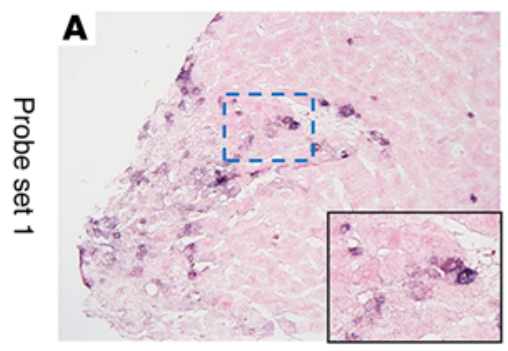

DNase I

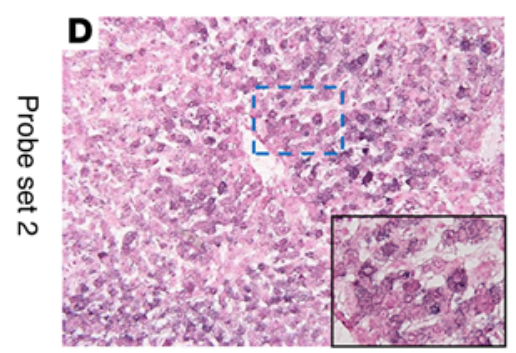

No treatment

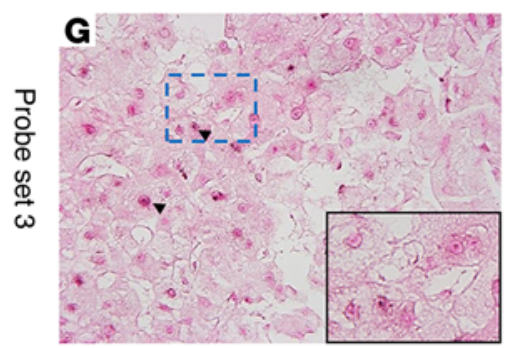

RNase $\mathrm{A} / \mathrm{H}+\mathrm{PSD}$

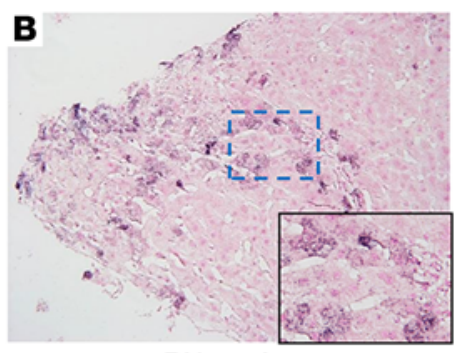

RNase A

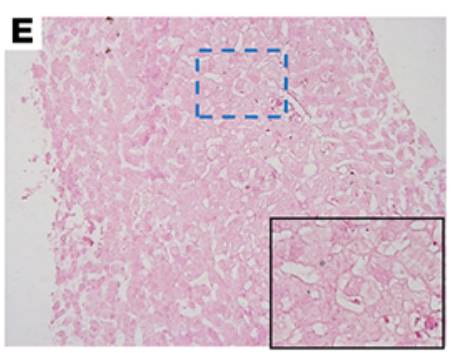

DNase I

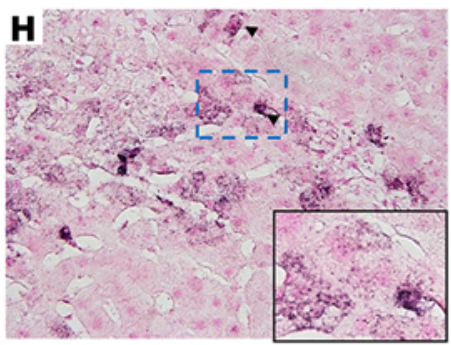

DNase I

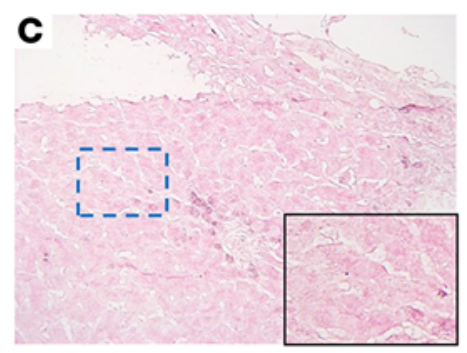

DNase I + RNase A

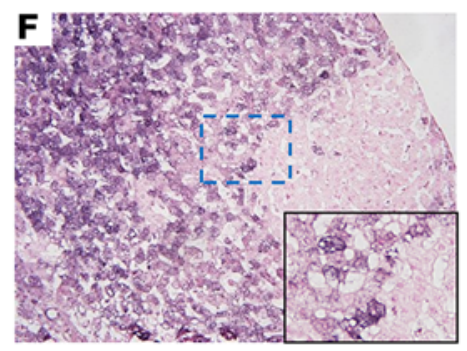

RNase A

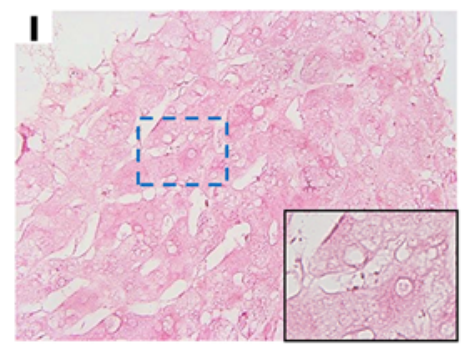

RNase A + DNase I

Figure 2. Validation of the specificity of the strand-specific probe sets design. Tissue sections from CHB patients were pretreated with the indicated nuclease and hybridized with the designated probe sets: (A-C) probe set 1 (original magnification, $\times 200$; insets, $\times 400$ ); (D-F) probe set 2 (original magnification, $\times 200$; insets, $\times 400$ ); (G-I) probe set 3 (original magnification, $\times 400$; insets, $\times 600$ ). After hybridization and signal amplification, NBT/BCIP was used for colorization, followed by counterstaining with nuclear fast red.

Subcellular localization of HBV DNA signal is highly related to its replication status. Having established our methodology, we then evaluated the localization pattern of HBV DNA in tissue sections from $47 \mathrm{CHB}$ patients with differing serological and virological status. We observed that, using probe set 2 , which hybridizes to the minus strand of HBV DNA, the colorized dots could reside in different subcellular locations, i.e., cytoplasmic (Figure 4A), nuclear (Figure 4B), or mixed. We reasoned that this differential pattern is mainly dictated by the robustness of viral replication. Indeed, comparison of serum HBV DNA levels in patients with distinct intracellular HBV DNA patterns showed a clear difference (cytoplasmic: median $=1.6 \times 10^{7}$ copies $/ \mathrm{ml}$; nuclear: median $=1.0$ $\times 10^{4}$ copies $/ \mathrm{ml} ; P<0.0001$, Mann-Whitney $U$ test; Figure $\left.4 \mathrm{C}\right)$. When grouped with e antigen status, although not statistically significant $(P=0.08$, Fisher's exact test), we observed a moderately higher proportion of cytoplasmic localization patterns in hepatitis B e antigen-positive (HBeAg-positive) patients (71.9\%) than in HBeAg-negative patients (41.7\%, Figure 4D). These results demonstrated that the vigor of $\mathrm{HBV}$ replication directly influenced the localization pattern of HBV DNA.

Relationships between intrahepatic viral nucleic acids and surface antigen. We next sought to explore the possibility of combining the hybridization of HBV DNA, RNA, and cccDNA with immune detection of major viral antigens, which might yield additional information about their distribution patterns. To this end, we visualized HBV DNA and cccDNA, followed by immunohistochemical staining of HBsAg. To our surprise, our inspection of 38 double-stained sections led to the observation that intrahepatic accumulation of HBsAg (Figure 5, brown) was inversely correlated with viral DNA or cccDNA (Figure 5, blue-purple), as exemplified by double staining of adjacent sections from 3 patients with $\mathrm{CHB}$ (Figure 5, A and B, and Supplemental Figure 6, A-D). In magnified images (Figure 5, C and D), it was clear that a high level of HBsAg almost inevitably led to depletion of viral DNA and vice versa. The same pattern was observed in CHB patients with low serum HBV DNA levels (Supplemental Figure 6, E and F, serum viral load $1.0 \times 10^{3}$ copies $/ \mathrm{ml}$ ). Nevertheless, cytoplasmic rcDNA and nuclear cccDNA were largely within similar regions (compare Figure 4, A and B, and Supplemental Figure 5, A and B). Similar patterns were found if IHC of HBsAg was performed prior to ISH or by using a fluorescently labeled secondary Ab for visualization of HBsAg (Supplemental Figure 7, $n=16$ ). It should be noted that colocalization of $\mathrm{HBsAg}$ and HBV DNA and cccDNA was found, albeit infrequently, in a few specimens in which these colocalized hepatocytes seemed to represent a transitional stage (Supplemental Figure 6, G and 
A

Biopsy

- T5 Spel

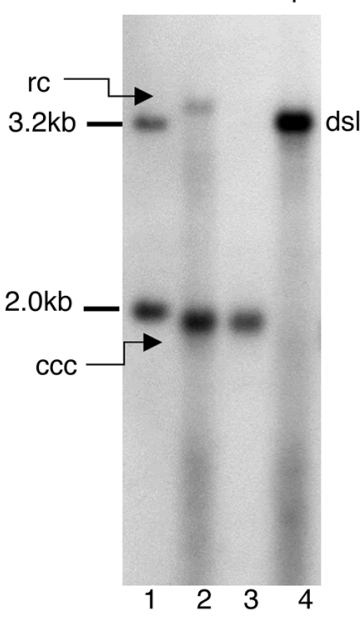

HepAD38

- T5 Spel

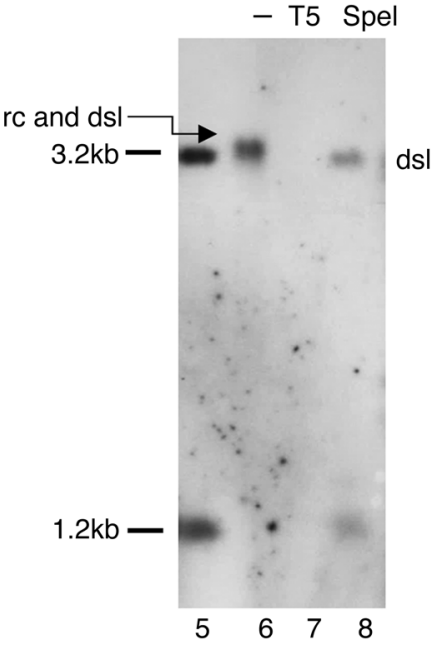

$\mathbf{B}$

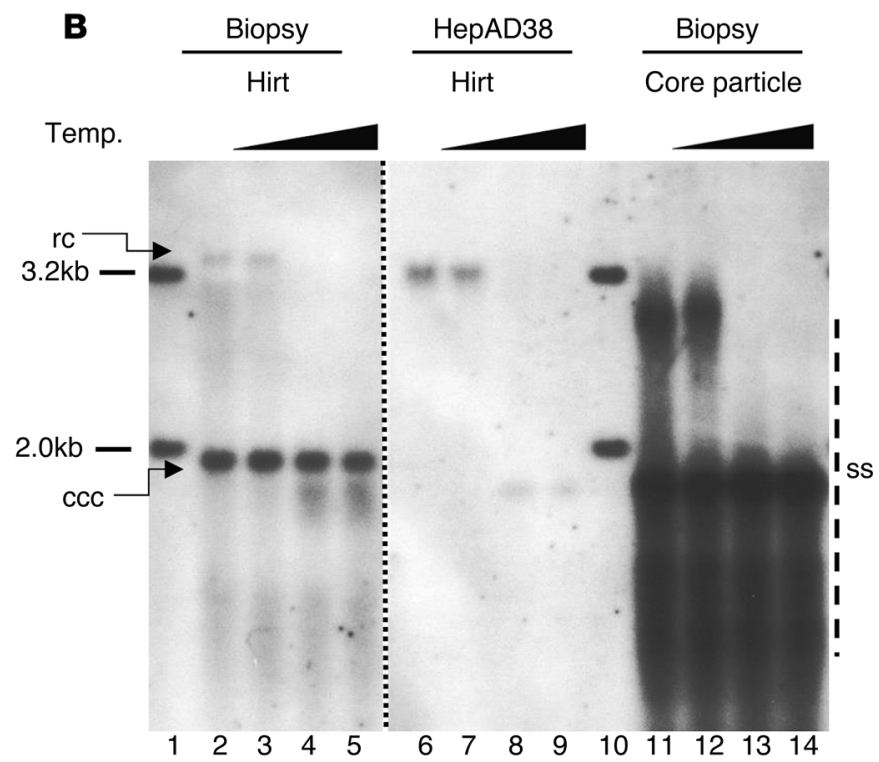

\section{C}

Biopsy M HepAD38

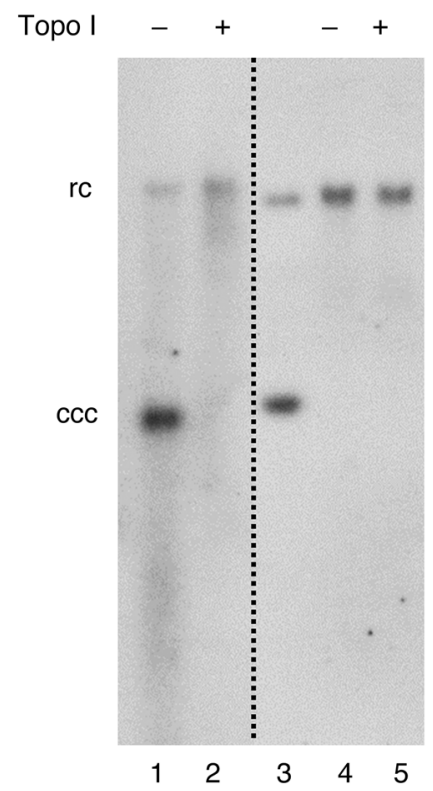

D

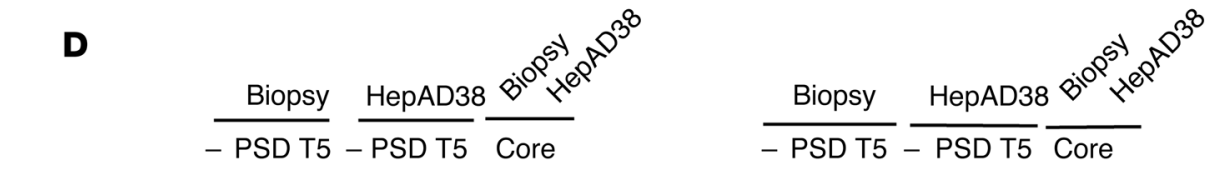

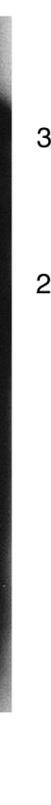

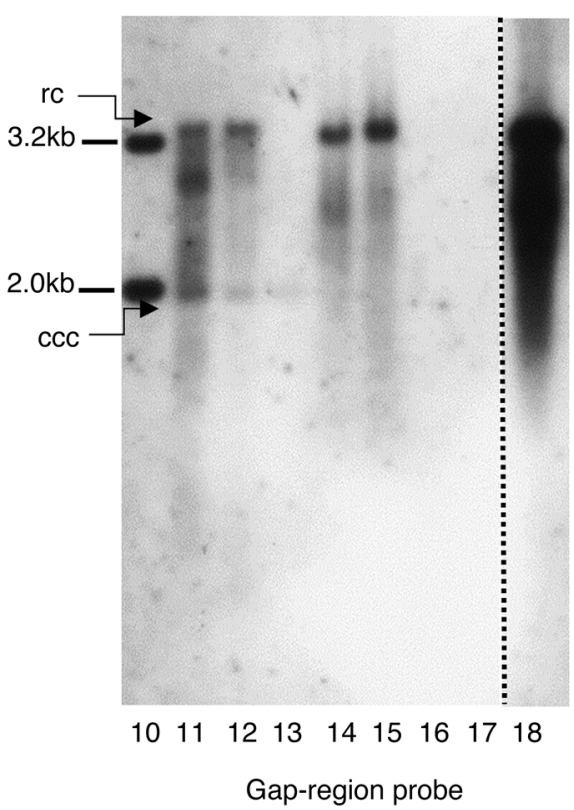

Figure 3. Southern blot analysis of protein-free and total DNA from liver biopsies. (A) Protein-free DNA from liver biopsies ( 5 mg) and HepAD38 cells $\left(1 \times 10^{7}\right.$ cells) was extracted using a modified Hirt's procedure. DNA was left untreated or digested with T5 exonuclease or Spel restriction nuclease, followed by Southern blotting using riboprobe 1. (B) Protein-free DNA from a liver biopsy and HepAD38, together with core particle DNA from a biopsy was left untreated (lanes 2, 6, and 11) or heated with $75^{\circ} \mathrm{C}$ (lanes 3, 7, and 12), 85 ${ }^{\circ} \mathrm{C}$ (lanes 4, 8, and 13), or $95^{\circ} \mathrm{C}$ (lanes 5,9 , and 14) for 5 minutes and subjected to Southern blot analysis using riboprobe 1. Temp., temperature. (C) Protein-free DNA from a liver biopsy and HepAD38 cells was left untreated or treated with topoisomerase I (Topo I) and subjected to Southern blot analysis using riboprobe 1. M, molecular marker (2.0 and $3.2 \mathrm{~kb}$ ). (D) Total DNA (lanes 2-7 and 11-16) and core particle DNA (lanes 8 and 9 and 17 and 18) from liver biopsies and HepAD38 cells was predigested with RNase A, RNase H alone (lanes 2, 5, 8, 9, 11, 14, 17, and 18), or in combination with PSD (lanes 3, 6, 12, and 15) or T5 exonuclease (lanes 4, 7, 13, and 16) for 1 hour at $37^{\circ} \mathrm{C}$. The treated DNA was hybridized with minus strand-specific riboprobe 1 (lanes 1-9) or gap region-specific riboprobe 2 (lanes 10-18). Images in B-D (right panels) were generated by joining noncontiguous lanes on the same blot. 

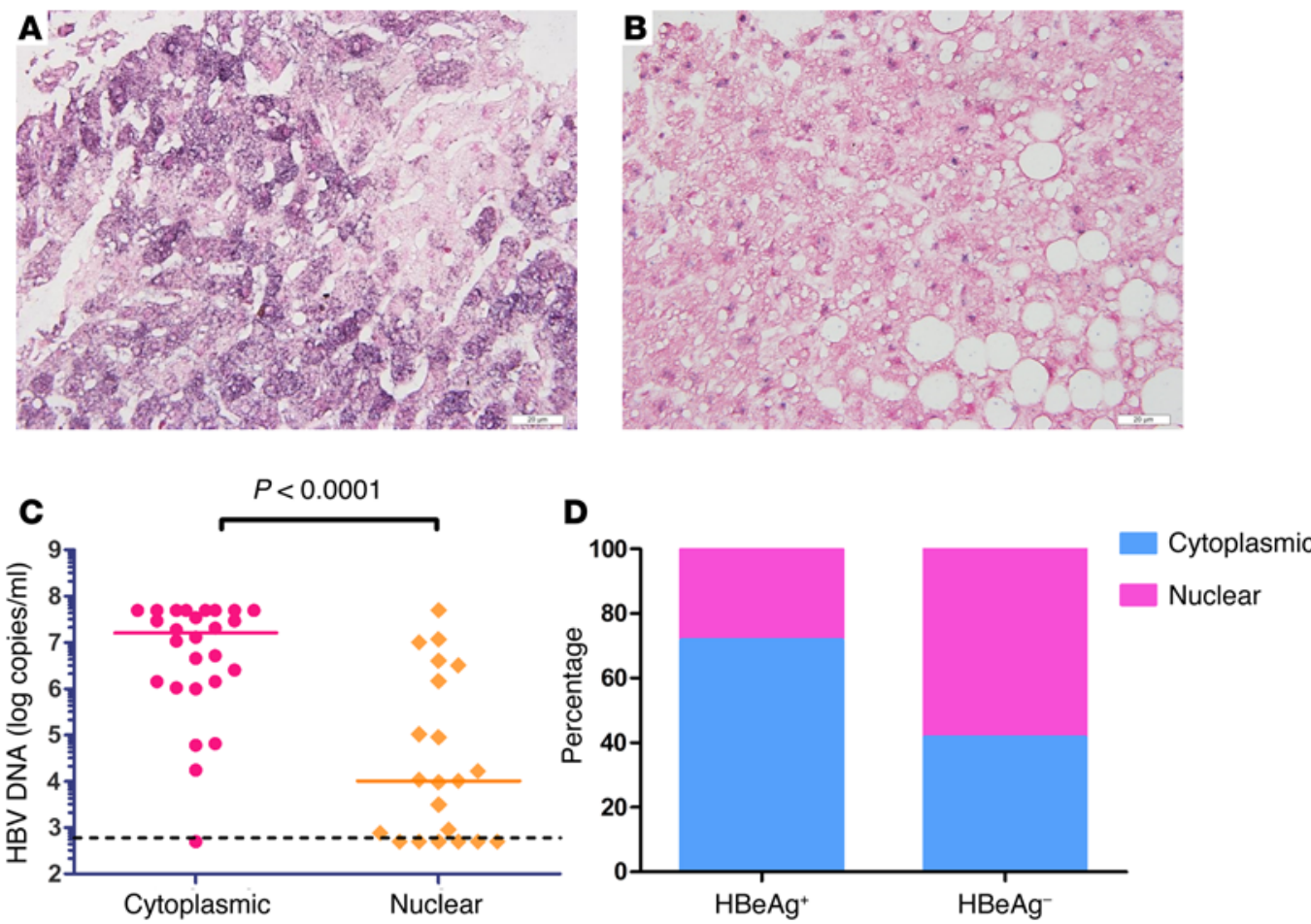

Figure 4. Subcellular localization of HBV DNA signal is highly related to its replication status. Representative cytoplasmic (A) and nuclear (B) staining pattern of HBV DNA. (C) Serum HBV DNA levels from cytoplasmic- $(n=26)$ and nuclear-localized ( $n=21$ ) groups were compared. The $P$ value was determined by a Mann-Whitney $U$ test. (D) Comparison of the percentage of cytoplasmic- and nuclear-localized samples from HBeAg-positive and -negative patients. patients with $\mathrm{CHB}$. It was intriguing to observe an even more complex pattern, in which HBV DNA was restricted to neither HBsAg- nor HBcAgexpressing cells (Figure 6, $\mathrm{E}$ and F, and Supplemental Figure 8, A-D). Rather, abundant intracellular surface antigen usually coincided with either no detectable or ultra-low levels of HBV DNA (Figure 6F, inset), consistent with the findings described above. Furthermore, in cells with nuclear localization of $\mathrm{HBcA}$, we observed very weak dots of nuclear cccDNA (see Figure $6 \mathrm{~F}$ and Supplemental Figure 8, B and D, insets), although cytoplasmic accumulation of DNA was also documented occasionally (Supplemental Figure 8E, arrows). In hepatocytes with cytoplasmic HBcAg, which is a less common distribu-
$\mathrm{H}$, arrows). More surprisingly, when combining HBV RNA visualization with HBsAg detection, a lack of colocalization was also found, although this trend was less prominent than that of $\mathrm{HBV}$ DNA and HBsAg (Figure 5, E and F, $n=8$ ). Probe set 1 (Figure $5 \mathrm{E}$ ), which exclusively targets pgRNA, and probe set 3 (Figure $5 \mathrm{~F}$ ), which also binds to subgenomic RNAs, yielded similar patterns. These observations seemed to suggest that intracellular accumulation of surface antigen did not require higher levels of equivalent mRNAs.

Highly complex distribution of $\mathrm{HBs} A g, \mathrm{HBcAg}$, and viral DNA. To further characterize the spatial relationship among major viral antigens (HBsAg and $\mathrm{HBcAg}$ ) and HBV DNA, we first visualized HBsAg and HBcAg using double immunohistochemical staining in sections from $25 \mathrm{CHB}$ patients (HBsAg and HBcAg double-positive as previously diagnosed by IHC). Again, we observed a low level of colocalization between $S$ and core antigens in these samples. As shown in a typical section (Figure 6, A and B), most nuclear core antigen-positive cells did not contain cytoplasmic surface antigen (colocalization index, 8.9\%). To exclude the possible masking effect made by IHC colorization, we performed double immunofluorescence staining of a section from the same specimen and analyzed the results using TissueFAXS scanning software. As shown in Figure 6C, the scatterplot confirmed the largely separate nature of these 2 antigens. In addition, we surveyed and calculated the colocalization indices of these 25 samples, which yielded a median rate of $6.67 \%$ (Figure 6D).

Next, we moved forward to integrate the HBsAg and HBcAg double IHC with ISH of HBV DNA using liver sections from 33 tion pattern, HBV DNA more frequently colocalized with core antigen (Supplemental Figure 8F, see arrows and note the faint blue-purple signal accompanying the red signal). In general, a greater colocalization was observed between $\mathrm{HBcAg}$ and $\mathrm{HBV}$ DNA than that seen between HBsAg and HBV DNA.

Relationships between HBV DNA and cccDNA. Although previous results indicated that HBV rcDNA colocalized with cccDNA, it was still not clear whether the amount of cccDNA was proportional to cytoplasmic DNA. To this end, we further visualized HBV DNA and cccDNA in adjacent sections of 5 liver specimens. As shown in Figure 7A, the amount of HBV DNA showed a high level of variability. Some regions showed intense cytoplasmic HBV DNA staining, while weak signals in cells were almost exclusively restricted to the nuclei. On the other hand, the overall distribution of cccDNA showed much less fluctuation (Figure 7B). The difference in the amount of these 2 types of DNA is highlighted by the insets showing magnified images of the same area (Figure 7, A and B). These results clearly suggested that the nuclear pool of cccDNA was maintained in a relatively steady manner and was not dramatically affected by the cytoplasmic replicative intermediates.

The in vivo persistence of cccDNA after NA therapy. Our method, which is able to visualize cccDNA in clinical liver specimens, provided us with an opportunity to study the in vivo status of cccDNA with single-cell resolution as well as its physiological half-life during NA therapy. Nine pairs of serial biopsies taken from patients with $\mathrm{CHB}$ before and 1 year after adeforvir therapy were used to probe the distribution of HBV DNA and cccDNA. Although significant person-to-person variations existed, we observed a general 

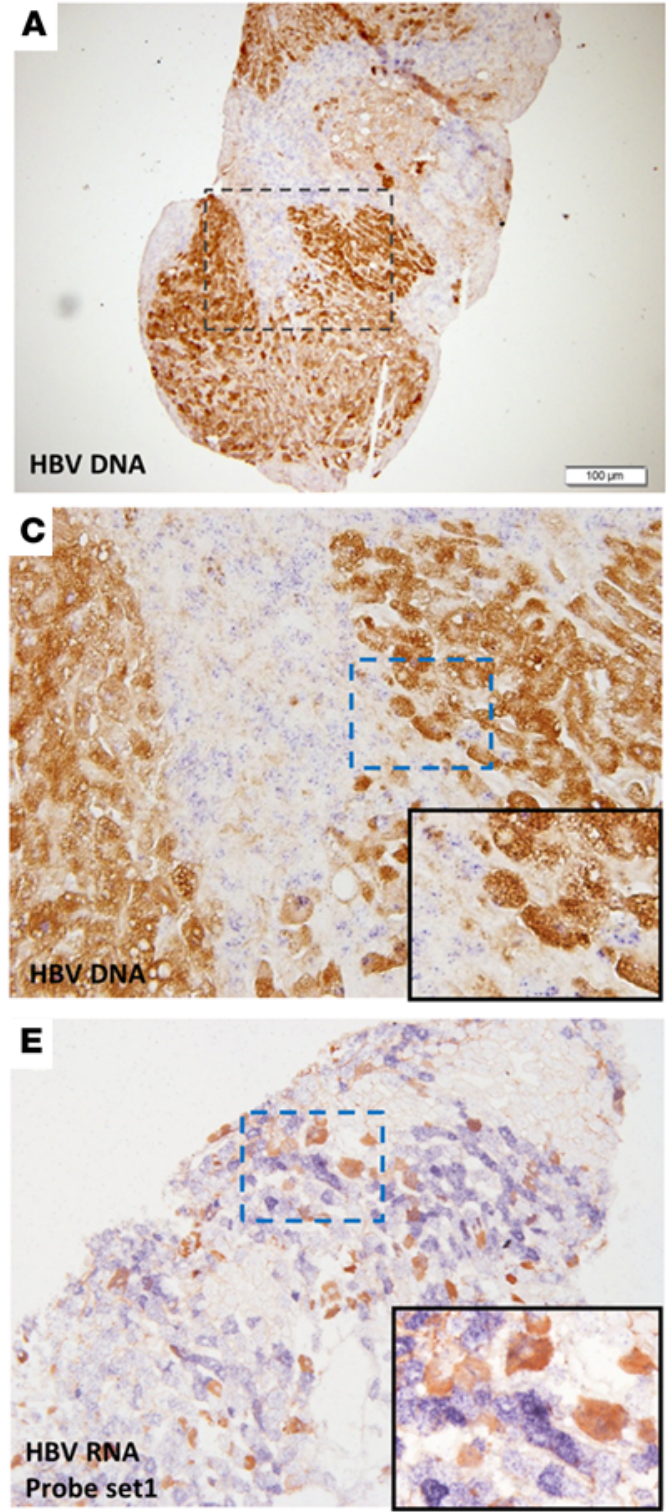
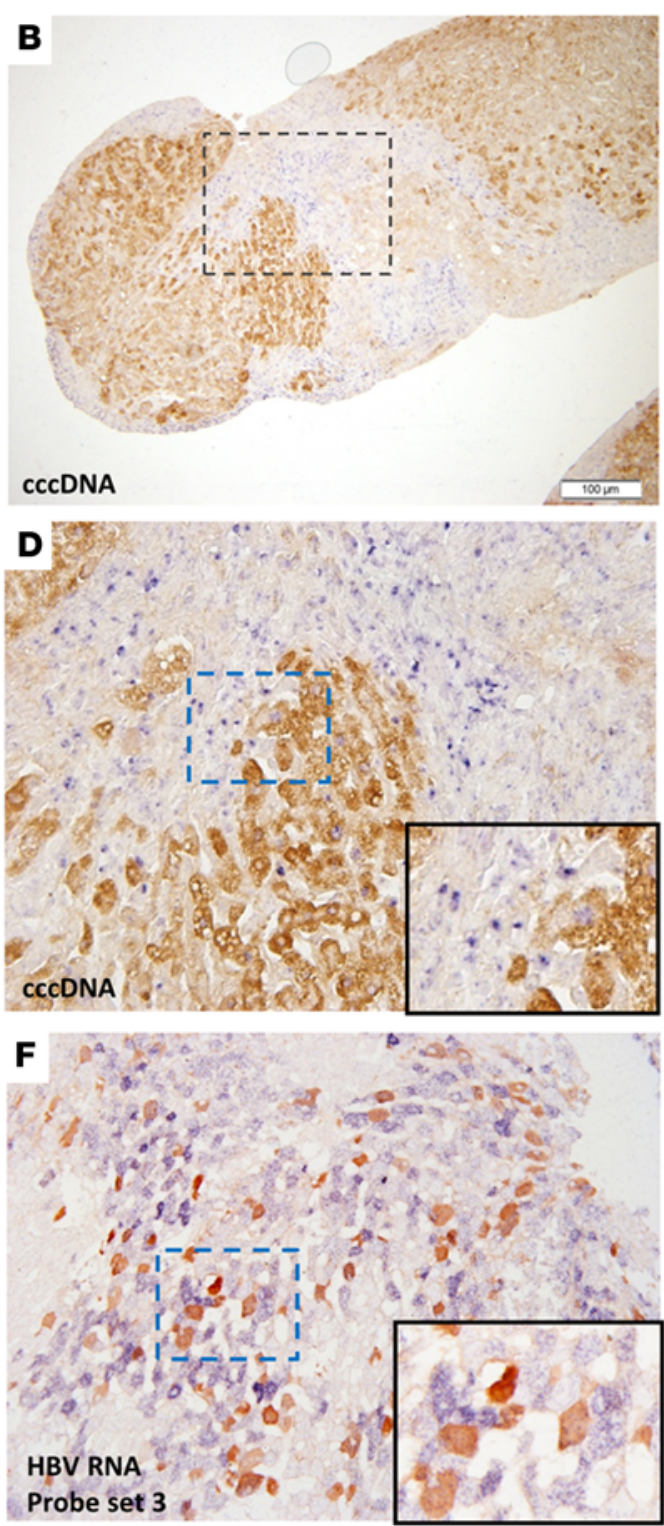

Figure 5. Spatial relationship between intrahepatic viral nucleic acids (HBV DNA, RNA, and (ccDNA) and surface antigen. Probe set 2 or 3 was hybridized with HBV DNA ( $\mathbf{A}$ and $\mathbf{C}$ ) or cccDNA (B and D), in conjunction with $\mathrm{HBsAg}$ IHC using adjacent sections from the same liver specimen. Images in $\mathbf{C}$ and $\mathbf{D}$ are higher magnifications of the boxed areas in $\mathbf{A}$ and $\mathbf{B}$, respectively, which show similar regions in adjacent slices. HBV RNA was visualized with either probe set $1(\mathbf{E})$ or probe set $3(\mathbf{F})$ in the context of HBsAg expression using adjacent sections from the same liver specimen. Blue-purple, DNA, cccDNA, or RNA; brown, HBsAg. Original magnification, $\times 100$ (A and $\mathbf{B})$, $\times 400$ (C and D), $\times 600$ (C and D insets), $\times 200$ (E and F) $\times 400$ ( $E$ and $\mathbf{F}$ insets). decline of cytoplasmically positive cells, whereas nuclear signals became dominant after 48 weeks of therapy (Figure 8, A and B, Supplemental Figure 9, and Supplemental Table 2). For HBV cccDNA, a general decline in signal intensities was found. However, it was evident that, qualitatively, the proportion of hepatocytes carrying nuclear cccDNA showed little change (Figure 8, C and D, Supplemental Figure 9, and Supplemental Table 2). For HBsAg IHC, although 4 patients showed marked depletion of HBsAg-positive rates, 4 patients did not show a noticeable difference, and 1 patient even showed an increased positive rate (Supplemental Table 2). Thus, we concluded that the intranuclear reservoir of HBV was not qualitatively purged in the slightest degree after 1 year of adeforvir treatment.

\section{Discussion}

Current knowledge of the formation and maintenance of HBV cccDNA. cccDNA plays a central role in establishing a persistent HBV infection. Its biogenesis and maintenance have been extensively stud- ied over the past 30 years. Pioneering work using the DHBV model has established that cccDNA is initially formed after viral entry and nuclear translocation (5). As the template for viral RNA synthesis, cccDNA is maintained via an intracellular recycling pathway that converts cytoplasmic rcDNA into a nuclear, polymerase-free, covalently closed circular form (2). The study of HBV cccDNA, however, is seriously hampered by the lack of a suitable model system. Despite these difficulties, some studies were performed to characterize the nuclear supercoiled HBV DNA using liver specimens from HBV-infected patients or HBV-infected chimpanzees $(9,10)$. These studies confirmed the overall features of cccDNA as observed in DHBV infection. However, lower cccDNA loads were found in human liver biopsies (0.1-1 cccDNA copy/cell) (3) compared with those detected in chronically infected ducks (11) and woodchucks (12). This discrepancy was suggested to be primarily determined by the virus, not the host, as evidenced by crossspecies transfection experiments (13). The detailed analyses of the key steps required for the formation of HBV cccDNA were mostly 

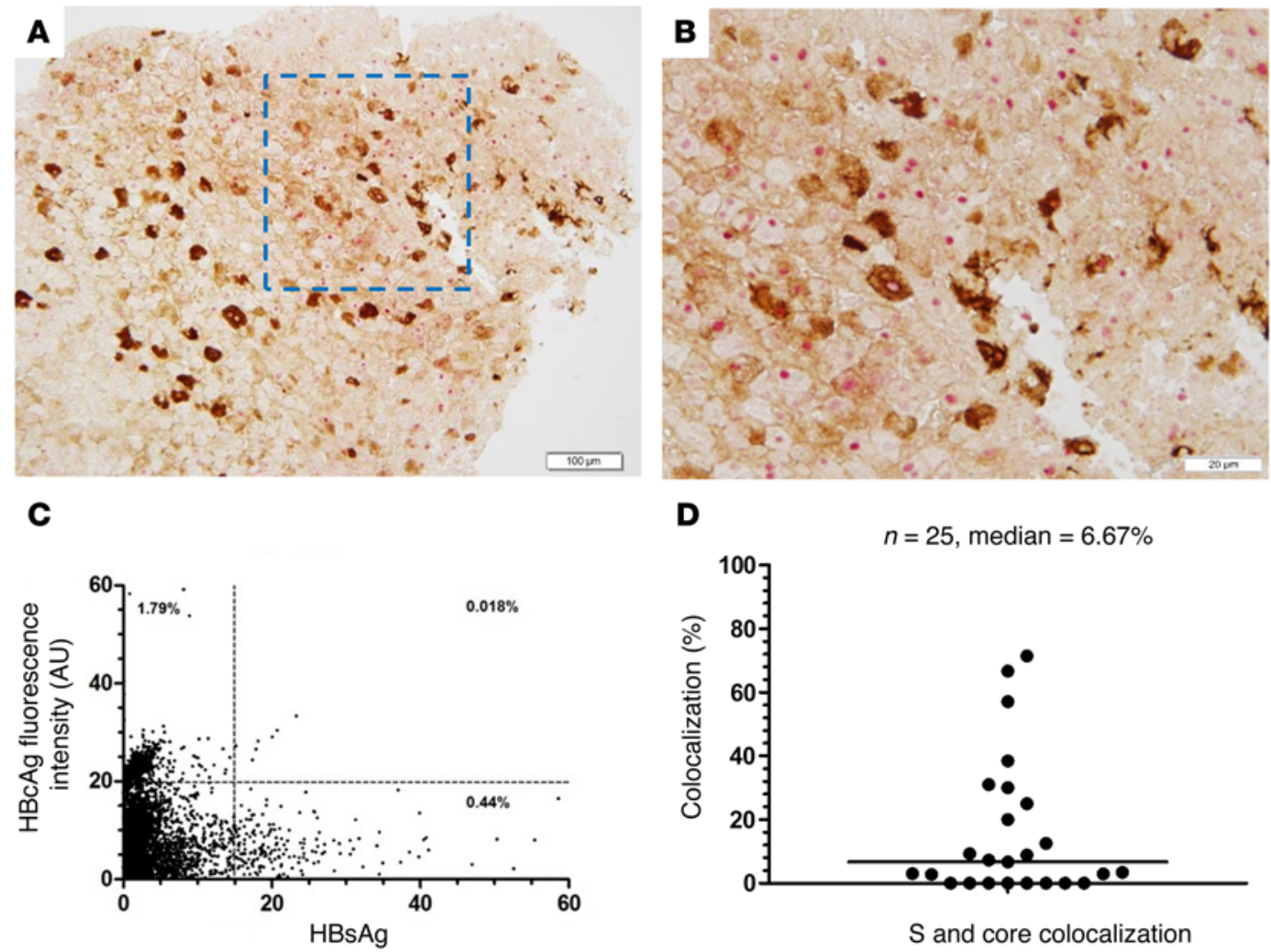

S and core colocalization
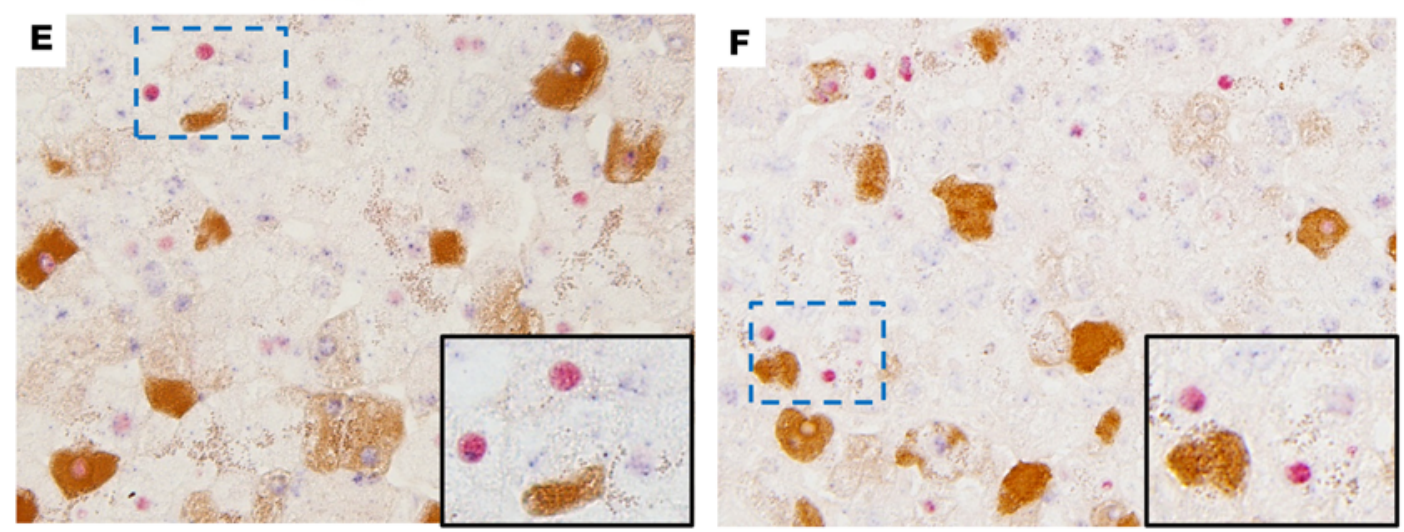

Figure 6. Highly complex distribution of $\mathrm{HBsAg}, \mathrm{HBcAg}$, and viral DNA. Liver sections from $\mathrm{CHB}$ patients were double stained with anti-HBsAg and anti-HBcAg, followed by visualization with $\mathrm{DAB}$ and permanent red, respectively. Results from a representative specimen are shown. Original magnification, $\times 200$ (A), $\times 400$ (B). The image in B is a magnification of the boxed area in A. Double immunofluorescence staining of $\mathrm{HBs} A g$ and $\mathrm{HBc} A g$ from the same specimen was performed and analyzed with TissueQuest. (C) Scatterplot showing the expression of HBsAg and HBcAg. (D) Colocalization indices from $25 \mathrm{HBsAg}$ and HBcAg double-positive specimens were calculated. (E and $\mathbf{F}$ ) ISH of HBV DNA with probe set 2, in conjunction with HBsAg and $\mathrm{HBCAg}$ double IHC. Original magnification, $\times 400$ and $\times 600$ (insets).

based on transient transfection or inducible initiation of replication in hepatoma cell lines $(6,7,13)$. These studies found that the amount of PF-rcDNA, as the precursor to supercoiled DNA, far exceeded the amount of cccDNA. This differed substantially from the findings of a previous report (9) and our observations using samples from CHB patients. Indeed, our Southern blot assay indicated the dominance of cccDNA over PF-rcDNA, as evidenced by its resistance to $\mathrm{T} 5$ exonuclease digestion and heat denaturation and also by its mobility shift after topoisomerase I treatment. It should be noted that normal human hepatocytes have a very slow multiplication rate (approximately 400 days), whereas hepatoma cells constantly divide. Meanwhile, it is well recognized that hepatocyte proliferation markedly reduces intrahepatic cccDNA loads in vivo (14). Thus, this layer of difference may contribute to the low efficiency of cccDNA formation in cell lines. Hence, in vivo data on the abundance and distribution of cccDNA would be instrumental for understanding its physiological status.

The merits of our ISH technique. Heterogeneity is an inherent feature of all multicellular organisms. Bulk measurements inform on the average level of a certain molecule but lose important spatial information (15). In this study, we sought to perform a purely observational study on the distribution of HBV nucleic 
A

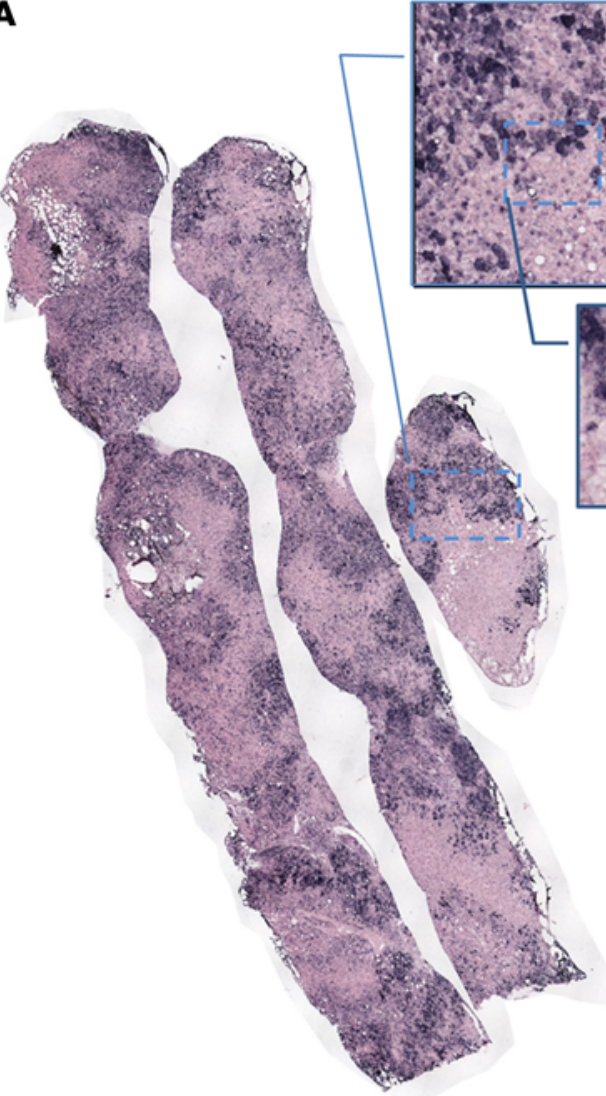

HBV DNA
B

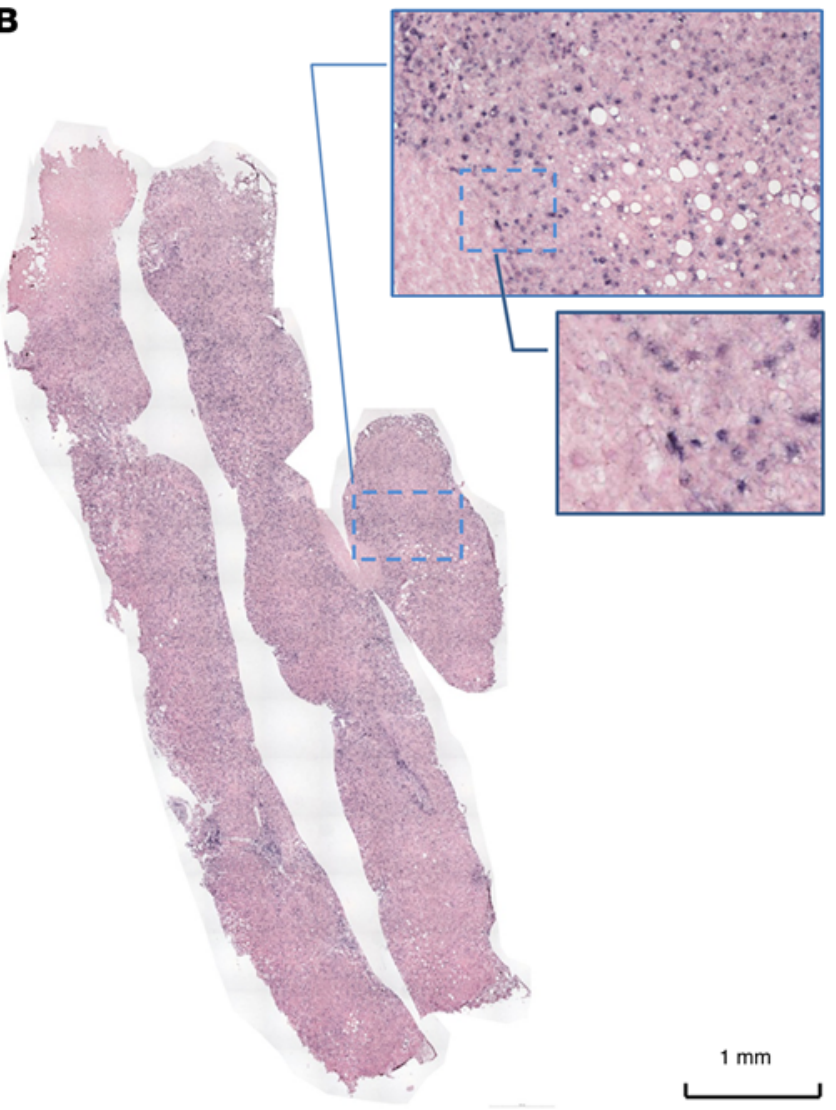

$\operatorname{cccDNA}$

Figure 7. Relationship between HBV DNA and cccDNA. Whole-tissue scan of HBV DNA (A) and cccDNA (B) ISH from adjacent sections of 5 CHB liver specimens. One pair of representative images is shown. The selected regions were serially magnified (original magnification, $\times 200$ and $\times 600$ ) in the insets.

acids (rcDNA, RNA, and, in particular, cccDNA) at single-cell resolution in liver specimens from patients with CHB. In retrospect, radioactively labeled HBV DNA probes had already been used in the early 1980s for in situ detection of HBV DNA $(16,17)$. Later studies turned to hapten-labeled probes to trace HBV DNA (18, 19). These studies generated important information regarding the relationship between the disease state and intrahepatic DNA localization. Nevertheless, the sensitivity of these assays was generally low, as evidenced by weak or no signal in HBeAg-negative specimens $(17,18)$. More important, the probes they used were incapable of distinguishing plus- and minus-strand DNA, let alone cccDNA. One report described ISH of cccDNA in HepG2-HBV cells using a plus strand-specific RNA probe (20), but it was not tested in liver tissues. Meanwhile, other researchers used PCR-based ISH to detect HBV DNA and RNA (21). However, the detection of viral nucleic acids in almost all hepatocytes raised concerns regarding the diffusion of amplified DNA to neighboring cells, which had been a major issue with in situ PCR (22). Recently, a method using rolling circle amplification (RCA), combined with in situ PCR, was reported to detect HBV cccDNA (23). However, RCA reaction for the detection of DNA in formalinfixed tissues would presumably encounter serious problems, since cross-linked histones or other cccDNA-binding proteins would hinder effective amplification. In addition, the diffusion problem with in situ PCR would also compromise the specificity of this methodology (22).

In this study, we exploited and modified the ViewRNA technology, making it capable of visualizing RNA and DNA. The ViewRNA technology uses the branched DNA (bDNA) amplification scheme, which was reported to achieve single-copy gene detection under optimal conditions, while preserving high specificity $(24,25)$. Most important, this methodology does not rely on enzyme-based nucleic acid amplification, which obviates complications such as cross contamination or diffusion of amplified products. Another strength of our methodology is that, by careful design of probe sets combined with suitable nuclease pretreatment, we were able to specifically visualize the localization of HBV DNA (minus or plus strand), HBV RNA (pgRNA or total HBV RNA), and cccDNA, either alone or in combination with IHC or immunofluorescence. Thus, this platform provided us with the capability to detect virtually all types of $\mathrm{HBV}$ nucleic acids in the context of viral or host antigen. A series of Southern blot analyses verified the ultra-low level of crossreactivity of the gap region probe toward cytoplasmic core particle DNA. In addition, apart from the canonical cccDNA detected by the gap region probe, a relaxed form of nuclear protein-free DNA was also observed. This species was previously named "open circular" (26) or "relaxed circular" (5) DNA in DHBV studies. In human hepatoma cell lines, this species exists at a much higher level than 

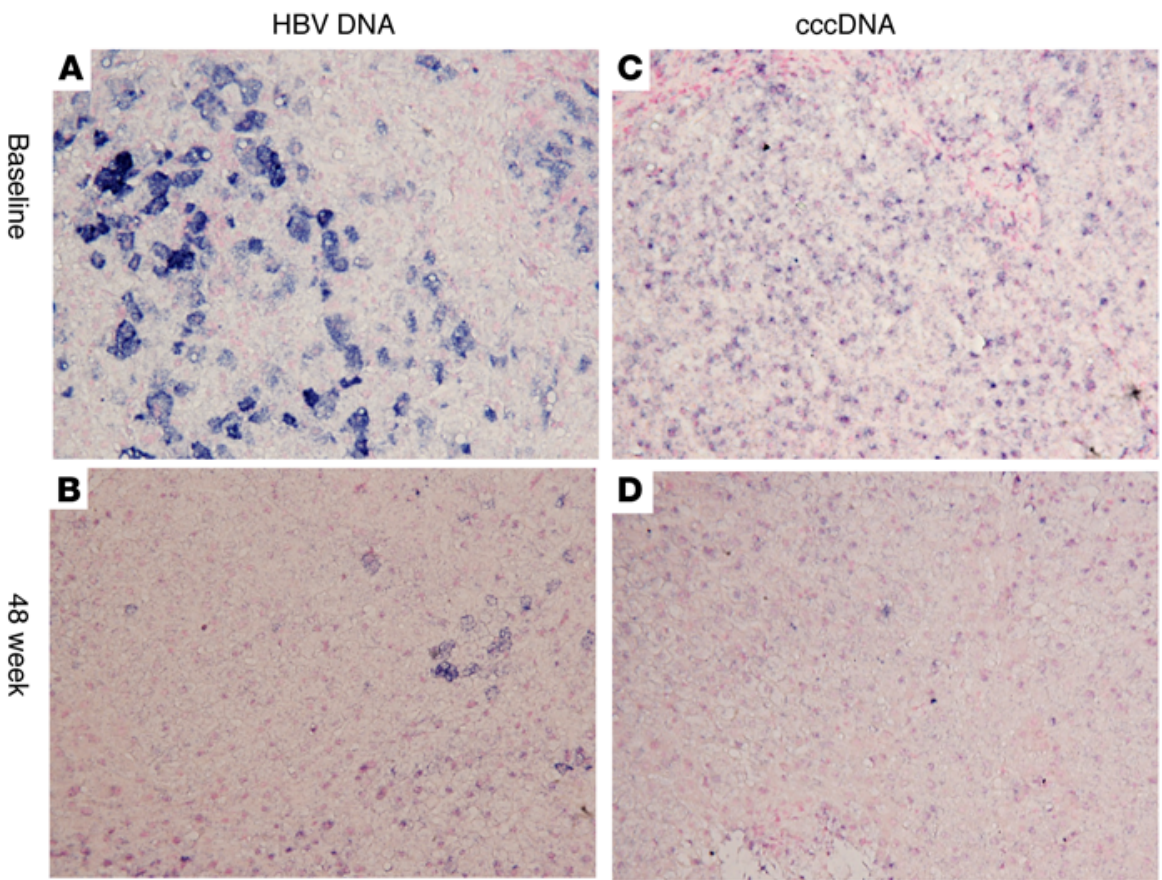

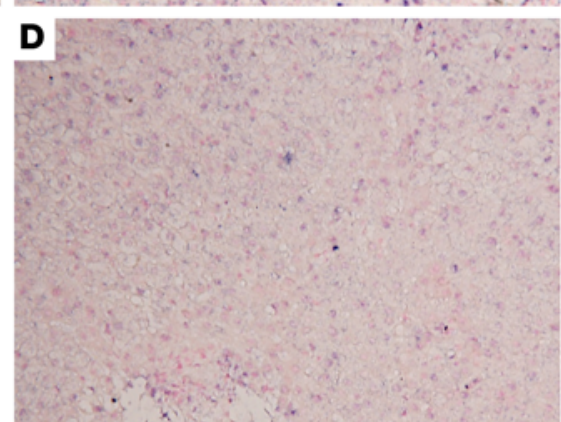

Figure 8. Distribution of HBV DNA and cccDNA before and after adeforvir therapy. Serial liver sections from 9 CHB patients before and 48 weeks after adefovir therapy were hybridized with probe set 2 or 3 to visualize HBV DNA (A and $\mathbf{B}$ ) or cccDNA (C and $\mathbf{D})$. Results from 1 representative patient (no. 3) are shown. Original magnification, $\times 200$.

cytoplasmic nucleocapsids exclusively into mature virus" (5). What we observed in this study indicated that this negative feedback control by surface antigen did not occur in a uniform manner but rather at the single-cell level. Indeed, on rare occasions, the observed interim state in a small percentage of hepatocytes (Supplemental Figure $6, \mathrm{G}$ and $\mathrm{H}$ ) suggested that transitions occurred between these stages. In addition, given the previous knowledge gained from the DHBV model, we inferred that HBsAg enrichment might precede nucleic acid accumulation during the natural infection

does cccDNA and contains a defined plus-strand end $(6,7)$. However, in the DHBV- or HBV-infected liver, no consensus has been reached as to whether it is a randomly nicked form of cccDNA generated during the purification procedure or a precursor to mature cccDNA, as reported in HepAD38 cells. In any case, our ISH results indicated that both supercoiled and relaxed forms of protein-free HBV DNA represented the nuclear genetic reservoir for HBV.

Relationships between intrahepatic surface antigen and viral nucleic acids. The application of this technique in clinical specimens yielded unexpected findings. The localization of intrahepatic HBV DNA and cccDNA inversely correlated with HBsAg. This seemingly counterintuitive result is actually theoretically plausible. Studies of DHBV cccDNA formation demonstrated that ablation of surface antigen expression dramatically increases cccDNA copy numbers per nucleus (27). Similarly, ablation of HBsAg also increased intranuclear HBV cccDNA formation, although to a lesser extent (7). Detailed examination using large (L), middle $(\mathrm{M})$, and small (S) envelope protein mutant constructs suggested that $\mathrm{L}$ and $\mathrm{M}$ proteins were mainly responsible for this phenomenon (28). In liver tissues from CHB patients, intrahepatic HBsAg levels showed an inverse correlation with serum HBsAg titers (29). Immunoblotting of $\mathrm{L}, \mathrm{M}$, and $\mathrm{S}$ proteins in paired liver and serum samples indicated that $\mathrm{L}$ protein is the major intrahepatic surface antigen species, whose accumulation in the cytoplasm results in a ground-glass morphology (30). It could be actively secreted only when vigorous viral replication took place (31). Given these findings, our observations seemed to suggest that HBsAg-high and HBV DNA-high hepatocytes reflected different stages in the HBV life cycle. In fact, as early as 1986, Tuttleman and colleagues had proposed that "negative control over the conversion of rcDNA into cccDNA would be effected through the production of a viral gene product whose concentration in the cell is dependent on the size of the cccDNA pool. This gene product would then block further synthesis of cccDNA directly or would direct assembly of process. As such, the cell-autonomous transition from the HBsAgrich stage to the DNA-rich stage resulted in a bipartite pattern, as we observed in the infected liver tissues.

Localization of HBcAg in clinical HBV infection. The core proteins of HBV or other hepadnaviruses assemble into icosahedral nucleocapsids for encapsidation of rcDNA. In CHB infection, $\mathrm{HBcAg}$ can reside in the cytoplasm or nucleus. Detailed analysis of 598 liver specimens established that nuclear distribution of HBcAg usually correlated with an immune-tolerant state, whereas cytoplasmic distribution was associated with chronic active hepatitis (32). Studies using HBcAg-transgenic mice or cell lines stably expressing core protein revealed that the subcellular localization of nucleocapsid is cell-cycle regulated $(33,34)$. This theory is further supported by analysis of liver specimens from patients with CHB. Chu et al. demonstrated that cytoplasmic HBcAg frequently coincided with proliferating cell nuclear antigen (PCNA) positivity (35). Thus, it is conceivable that, in the liver, $\mathrm{HBcAg}$ can reside in the cytosol only when chronic inflammation and subsequent injuryinduced hepatic regeneration occur.

In this study, we showed that massive cytoplasmic HBV DNA was not necessarily accompanied by the accumulation of cytoplasmic core antigen. These results, however, should be interpreted with caution. Considering the extensive literature supporting the essential role of core encapsidation in viral DNA synthesis, it may be that the current IHC method for HBcAg detection has far lower sensitivity than our ISH method, which led to these observations. Indeed, several reports showed more significant cytoplasmic HBcAg staining using frozen sections or formalin-fixed tissues with an improved pretreatment protocol $(36,37)$. In addition, it was recently found that genome-free empty virions composed of core and surface proteins existed in patients' sera and far exceeded the number of complete virions (by more than 100-fold) (38). This suggests that active synthesis of core proteins occurs inside the hepatocytes but may not be appreciated using conventional 


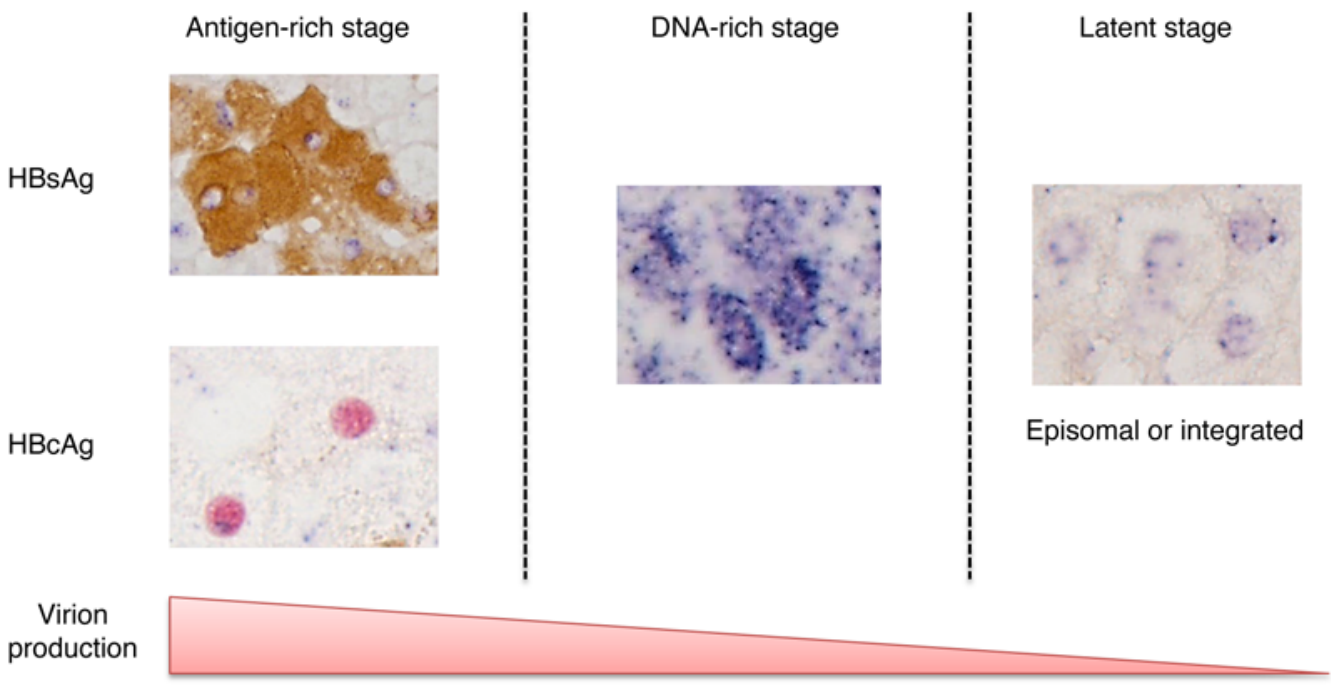

\author{
Figure 9. Three proposed \\ stages of the HBV life cycle at \\ the single-cell level. Typical \\ images were cropped and \\ show each stage. Original \\ magnification, $\times 600$.
}

cccDNA

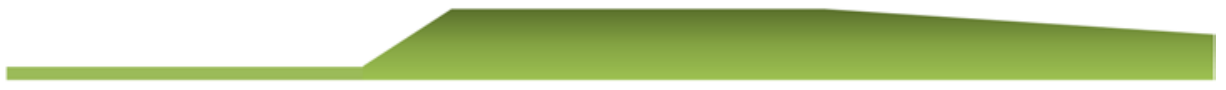

IHC methods. Further research is needed to optimize the detection method for $\mathrm{HBcAg}$ and reevaluate the relationship between $\mathrm{HBcAg}$ and HBV DNA.

Persistence of cccDNA after antiviral therapy. The half-life and distribution of cccDNA, which constitute the molecular basis of HBV persistence, have been a central problem in clinical HBV research. Previous clinical studies mainly used quantitative PCR assays capable of differentiating cccDNA from rcDNA to estimate the amount and clearance rate during antiviral therapy $(3,4)$. The results in these studies showed a cccDNA decline of approximately $1 \log _{10}$ copies per cell after 48 weeks of NA treatment (4). In this report, we provided another aspect of cccDNA decline by directly visualizing it in liver tissues. In $9 \mathrm{CHB}$ patients receiving adefovir therapy, we observed a marked decrease of intrahepatic HBV DNA. However, there was little difference in the prevalence of cccDNA, although the staining intensities declined marginally. Our data confirmed the findings of previous clinical studies $(3,4)$ regarding the resistance of cccDNA to commonly used therapies. Moreover, we observed this persistence at the single-cell level, i.e., a decline, but not a disappearance, of supercoiled DNA in almost all infected cells after 1 year of therapy.

Conclusion. It is widely accepted that, at the individual level, $\mathrm{CHB}$ infection can be categorized into immune-tolerant, clearance, control, and escape phases (2). By analogy, on the basis of the existing literature and data obtained from this study, we propose that, at the single-cell level, 3 distinct stages of HBV replication exist (Figure 9). The first is the antigen-rich stage, with abundant HBsAg or HBcAg levels. HBsAg accumulation is accompanied by ultra-low levels of cccDNA (presumably at single-digit copies/cell levels), while HBcAg-rich cells may harbor greater amounts of cccDNA. The second is the DNA-rich stage, in which large amounts of cytoplasmic replicative intermediates are accumulated, but without detectable viral antigens. In the meantime, the intranuclear pool of cccDNA begins to build up. The third is the latent stage, in which viral antigens and replicative intermedi- ates are hardly detectable, while HBV DNA remains in the nucleus - either in the form of episomal cccDNA (predominant in CHB) or as integrated DNA (more prevalent in HBV-related advanced cirrhosis and hepatocellular carcinoma), with no or very low-level replication - and survives long-term antiviral therapy. The tripartite stages might reflect the smart tactics used by this cunning virus in a changing cellular environment, which, in effect, results in a gradual transition from vigorous virion production to active stockpiling of genome copies and nuclear cccDNA reserves. This process helps the virus to maintain its occupancy of host cells, while avoiding excessive antigen-specific immune responses. It would be most desirable to observe longitudinal liver specimens from animals experimentally infected with HBV to elucidate the exact sequence and magnitude of these stages during the entire infection process.

Our study also has its limitations. Although integration is not strictly required for the HBV life cycle and was not detectable by Southern blot analysis in CHB patients without cirrhosis or hepatocellular carcinoma (39), hepatocyte clones harboring an integrated HBV sequence do exist (40), and our cccDNA-specific probe set was unable to differentiate these events from episomal DNA. Second, since our probe sets were designed for the genome sequence of subtype $\mathrm{C} \mathrm{HBV}$, the sensitivity of our assay was influenced by sequence complementarity between viral and probe sequences. Further development is needed to achieve the detection of all genotypes using type-specific probe sets. In addition, enzyme-catalyzed fluorogenic methods with a sensitivity close to that of Nitroblue tetrazolium and 1,5-bromo-4-chlooro-3-indolylphosphate (NBT/BCIP) need to be developed to investigate the fine localization of viral replication in the context of viral or host proteins. Nevertheless, with reasonable assay optimization and standardization, our ISH assay has the potential to be applied as a routine pathological examination complementary to conventional $\mathrm{HE}$ staining and IHC and to provide much more comprehensive virohistological data for clinicians. 


\section{Methods}

Patients and specimens. A total of 164 individuals, including $160 \mathrm{CHB}$ patients and 4 control patients (1 HBV-negative, $1 \mathrm{HCV}, 1$ druginduced hepatitis, and 1 autoimmune hepatitis) were enrolled. Among these, we performed longitudinal observations of tissue sections from 9 CHB patients who underwent 1 year of adefovir therapy. The detailed clinical data (baseline and 48 weeks after therapy) for these 9 patients are provided in Supplemental Table 2. The clinical information for the other 155 patients is provided in Supplemental Table 1.

The percutaneous liver biopsy procedure was performed using needles with a 1-mm inner diameter. The liver specimens obtained were usually more than $1.2 \mathrm{~cm}$ long and separated into 2 parts and preserved differently (one with $10 \%$ neutral formalin and the other with RNAlater from Life Technologies). The blood samples from enrolled patients were tested for HBsAg and HBeAg using an Abbott AxSYM HBsAg Assay (normal: 0-2S/N) and an HBeAg 2.0 MEIA kit (normal: 0-1.0S/CO) (Abbott Laboratories) and for viral load using an HBV DNA quantitative real-time PCR kit (QIAGEN).

ISH of viral RNA, rcDNA, and cccDNA. The hybridization and amplification procedures were performed using the ViewRNA ISH Tissue Assay (Affymetrix). The protocol was extensively modified to detect various types of viral nucleic acids. The ViewRNA assay is based on bDNA amplification technology, which realizes remarkable signal amplification, while preserving high specificity. Although this product was developed solely for RNA detection, it can also be used to detect DNA (25). All probe sets were custom synthesized by Affymetrix using sequences from an isolate (GenBank: AB675675, genotype $C$, most prevalent in eastern China), except for HCV (VF1-10121, target sequence JFH-1 NS3-NS5B region). Probe sets used to detect HBV DNA and cccDNA in HepAD38 cells were separately synthesized in order to match the HBV sequence.

The general procedures for tissue pretreatment, hybridization, and signal amplification are shown in Supplemental Figure 1. The tissue sections were routinely dewaxed and rehydrated, followed by antigen retrieval with sodium citrate buffer $\left(\mathrm{pH} 6.0,90^{\circ} \mathrm{C}-95^{\circ} \mathrm{C}\right.$ for 20 minutes), protease digestion (provided in the VeiwRNA ISH tissue assay kit, 300 -fold dilution for 10 minutes at $37^{\circ} \mathrm{C}$ ), and refixation with $3.7 \%$ neutral formaldehyde for 5 minutes. For HBV DNA hybridization, probe set 2 , which binds to the minus strand of the HBV replicative intermediate, was used. For HBV cccDNA detection, we used probe set 3 , which anneals to the gap region (plus strand) of HBV's partial double-stranded genome. Slides were pretreated with RNase A, RNase H (Fermentas, Thermo Fisher Scientific), and Plasmid-Safe DNase (Epicenter, Illumina) for 60 minutes at $37^{\circ} \mathrm{C}$ before hybridization. For HBV RNA detection, we used liver specimens protected with RNAlater, which preserves both RNA integrity and tissue morphology (41). Probe set 1 was used to detect pgRNA and probe set 3 to detect the sum of pgRNAs and subgenomic RNAs. To ensure removal of viral DNA, slides were predigested with RNase-free DNase I (Takara) for 60 minutes at $37^{\circ} \mathrm{C}$. After pretreatment and suitable nuclease digestion, slides were re-fixed with $3.7 \%$ neutral formaldehyde for 5 minutes and rinsed with deionized water. Probe sets were diluted 100 -fold into hybridization buffer ( $2 \times$ SSC, $10 \%$ dextran sulfate, $0.01 \%$ sheared salmon sperm DNA, $0.02 \%$ SDS, and $50 \%$ formamide) and added onto the sections. Coverslips were applied and the edges sealed with rubber cement. Slides were preheated at $95^{\circ} \mathrm{C}$ for 10 minutes and then cooled to $40^{\circ} \mathrm{C}$ overnight. Subsequent washing and bDNA amplification were performed as described by the ViewRNA ISH tissue assay manual (Panomics, Affymetrix). Finally, sections were stained with NBT/BCIP (Roche) in developing solution at $37^{\circ} \mathrm{C}(2$ hours to overnight), followed by counterstaining with nuclear fast red. In some instances, the counterstain was omitted to avoid masking of weak signals. Slides were dehydrated and mounted with UltraClear (Baso). To confirm the specificity of the hybridization signal, rigorous controls were performed in parallel, which included: (a) sections from HBV-negative individuals (for whom HBsAg, antiHBsAg, HBeAg, anti-HBeAg, and anti-HBcAg were all negative); (b) HBV-positive sections hybridized without probe or with HCV probe; (c) rat kidney sections hybridized with HBV probes; and (d) sections from patients with $\mathrm{HCV}$ or autoimmune or drug-induced hepatitis hybridized with probe sets 2 and 3 .

IHC and immunofluorescence staining. For IHC, sections were routinely dewaxed and rehydrated. After heat-induced antigen retrieval in sodium citrate ( $\mathrm{pH}$ 6.0) buffer, sections were incubated with a primary $\mathrm{mAb}$ against $\mathrm{HBsAg}$ (clone ZCH16; Life technologies) and rabbit polyclonal anti-HBcAg Abs (Dako). After washing, the polymer detection system (Polink-1 HRP; GBI Labs) was incubated for 30 minutes at room temperature and developed with DAB. For double staining of HBsAg and $\mathrm{HBcAg}$, a 2-plex polymerbased detection kit (Polink DS-MR; GBI Labs) was used. HBsAg and $\mathrm{HBcAg}$ were sequentially developed with $\mathrm{DAB}$ and permanent red, respectively. Fluorescent double staining of $\mathrm{HBsAg}$ and $\mathrm{HBcAg}$ was also performed using Alexa Fluor 488-conjugated goat anti-mouse IgG and Alexa Fluor 594-conjugated goat anti-rabbit IgG (Molecular Probes, Life Technologies), followed by counterstaining with Hoechst 33342 (Thermo Fisher Scientific).

Combined IHC and ISH. Serial IHC and ISH assays were performed for simultaneous detection of viral antigen and nucleic acid. For most slides, Ab staining and colorization were performed following the development of ISH signals. However, we found that these 2 steps could be exchanged without a significant effect on performance.

DNA extraction and Southern blot analysis. Core particle DNA from HepAD38 cells was extracted as previously described (7). Total DNA extraction from liver biopsies was performed using a PureLink Genomic DNA Kit (Life Technologies). Southern blot analysis was carried out using digoxingenin-11-UTP-labeled riboprobes generated by in vitro transcription using the DIG Northern Starter Kit (Roche Life Science). Two riboprobes were synthesized to detect HBV DNA and cccDNA, respectively. Riboprobe 1 targets the minus strand of HBV DNA (nt 900-1900, reference sequence: AB675675). Riboprobe 2 targets the gap region (plus strand, nt 500-1590, reference sequence: AB675675) of HBV rcDNA. For total DNA from liver biopsies, approximately $2 \mu \mathrm{g}$ total DNA was left untreated or coincubated with T5 exonuclease or topoisomerase I (New England BioLabs) and loaded on $1.2 \%$ agarose gel. For detection of cccDNA, after electrophoresis, gels were depurinated by $0.2 \mathrm{M}$ hydrochloride, followed by alkaline denaturation and neutralization essentially as described previously (42). DNA was transferred onto a positively charged nylon membrane (Roche Life Science) using a vacuum blotter (Bio-Rad). Subsequent procedures were performed according to the DIG Northern Starter Kit manual.

Image acquisition and processing. Light microscopic observation and image acquisition were mostly performed on an Olympus 
BX51 microscope equipped with a CCD (DP72) camera. Wholetissue scanning and fluorescence microscopy were performed on an automated scanning workstation (TissueFAXS; TissueGnostics), followed by analysis with TissueQuest (TissueGnostics). Quantification of Southern blot images was performed using Image Studio Lite software (LI-COR Biosciences).

Statistics. GraphPad Prism 5.0 (GraphPad Software) was used for most statistical analyses. A Mann-Whitney $U$ test was used to compare serum viral loads between 2 groups. Comparison between HBeAg-positive and -negative groups of the percentage of cytoplasmic or nuclear patterns of HBV DNA localization was made using Fisher's exact test. The colocalization index between 2 molecules at the single-cell level (e.g., HBsAg and $\mathrm{HBcAg}$ ) was calculated as $\mathrm{Co} / \mathrm{Min}(\mathrm{Ns}, \mathrm{Nc}) \times 100 \%$, where $\mathrm{Co}$ is the number of colocalized cells, Ns is the number of S-positive cells, and Nc is the number of core-positive cells. The ISH results were examined by 2 independent pathologists.

Study approval. Written informed consent was received from participants prior to their inclusion in this study and the collection of clinical samples. This study was conducted in compliance with the ethical guidelines of the 1975 Declaration of Helsinki and was approved by the ethics committee of the Shanghai Public Health Clinical Center.

\section{Author contributions}

$\mathrm{XZ}$ and $\mathrm{ZY}$ conceived and designed the study. XZ, YZ, WW, and LB conducted the experiments. WL, LC, and ZZ conducted the clinical study. XZ and WL analyzed the data. XZ, YZ and YF inspected the histological results. XZ, ZY, and ZZ wrote the manuscript.

\section{Acknowledgments}

We thank Zhuying Chen and Xiurong Peng for their excellent technical assistance and dedicated work in compiling the clinical data. We are grateful to Maya Kozlowski for her critical reading and editing of the manuscript.

This work was supported by the National Science and Technology Major Project of China (grant 2012ZX10002007-001); the National Natural Science Foundation (grant 81201278, 91542207); the National Key Basic Research Program of China (grant 2012CB519005); the National Health and Family Planning Commission (grant 201302010); and the Shanghai Science and Technology Commission (grant 15DZ2290200). This work was also supported by the Sino-German Transregional Collaborative Research Centre (grant 81461130019). The funders had no role in the study design, data collection and analysis, decision to publish, or preparation of the manuscript.

Address correspondence to: Zhenghong Yuan, Key Laboratory of Medical Molecular Virology at the School of Basic Medical Sciences, Shanghai Medical College, Fudan University, Shanghai 200032, China. Phone: 86.21.64161928; Fax: 86.21.64227201; E-mail: zhyuan@shaphc.org. Or to: Zhanqing Zhang, Department of Hepatology, Shanghai Public Health Clinical Center, Fudan University Caolang Road 2901, Shanghai 201508, China. Phone: 86.21.64161928; E-mail: doctorzzqsphc@163.com. Or to: Xiaonan Zhang, Research Unit, Shanghai Public Health Clinical Center, Fudan University Caolang Road 2901, Shanghai 201508, China. Phone: 86.21.64161928; E-mail: zhangxiaonan@shaphc.org.
1. 2015. Guidelines for the prevention, care and treatment of persons with chronic hepatitis B infection. World Health Organization. Geneva, Switzerland.

2. Dandri M, Locarnini S. New insight in the pathobiology of hepatitis B virus infection. Gut. 2012;61(suppl 1):i6-i17.

3. Werle-Lapostolle B, et al. Persistence of cccDNA during the natural history of chronic hepatitis $\mathrm{B}$ and decline during adefovir dipivoxil therapy. Gastroenterology. 2004;126(7):1750-1758.

4. Wong DK-H, et al. Reduction of hepatitis B surface antigen and covalently closed circular DNA by nucleos(t)ide analogues of different potency. Clin Gastroenterol Hepatol. 2013;11(8):1004-1010.

5. Tuttleman JS, Pourcel C, Summers J. Formation of the pool of covalently closed circular viral DNA in hepadnavirus-infected cells. Cell. 1986;47(3):451-460.

6. Gao W, Hu J. Formation of hepatitis B virus covalently closed circular DNA: removal of genomelinked protein. J Virol. 2007;81(12):6164-6174.

7. Guo H, Jiang D, Zhou T, Cuconati A, Block TM, Guo JT. Characterization of the intracellular deproteinized relaxed circular DNA of hepatitis B virus: an intermediate of covalently closed circular DNA formation. J Virol. 2007;81(22):12472-12484.

8. Miller RH, Marion PL, Robinson WS. Hepatitis $B$ viral DNA-RNA hybrid molecules in particles from infected liver are converted to viral DNA molecules during an endogenous DNA poly- merase reaction. Virology. 1984;139(1):64-72.

9. Miller RH, Robinson WS. Hepatitis B virus DNA forms in nuclear and cytoplasmic fractions of infected human liver. Virology. 1984;137(2):390-399.

10. Ruiz-Opazo N, Chakraborty PR, Shafritz DA. Characterization of viral genomes in the liver and serum of chimpanzee long-term hepatitis B virus carriers: a possible role for supercoiled $\mathrm{HBV}$ DNA in persistent HBV infection. JCell Biochem. 1982;19(3):281-292.

11. Zhang YY, Zhang BH, Theele D, Litwin S, Toll E, Summers J. Single-cell analysis of covalently closed circular DNA copy numbers in a hepadnavirus-infected liver. Proc Natl Acad Sci US A. 2003;100(21):12372-12377.

12. Dandri M, Burda MR, Will H, Petersen J. Increased hepatocyte turnover and inhibition of woodchuck hepatitis B virus replication by adefovir in vitro do not lead to reduction of the closed circular DNA. Hepatology. 2000;32(1):139-146.

13. Kock J, Rosler C, Zhang JJ, Blum HE, Nassal M, Thoma C. Generation of covalently closed circular DNA of hepatitis B viruses via intracellular recycling is regulated in a virus specific manner. PLoS Pathog. 2010;6(9):e1001082

14. Lutgehetmann $\mathrm{M}$, et al. In vivo proliferation of hepadnavirus-infected hepatocytes induces loss of covalently closed circular DNA in mice. Hepatology. 2010;52(1):16-24.

15. Itzkovitz S, van Oudenaarden A. Validating tran- scripts with probes and imaging technology. Nat Methods. 2011;8(4 suppl):S12-S19.

16. Gowans EJ, Burrell CJ, Jilbert AR, Marmion BP. Detection of hepatitis B virus DNA sequences in infected hepatocytes by in situ cytohybridisation. JMed Virol. 1981;8(1):67-78.

17. Burrell CJ, Gowans EJ, Rowland R, Hall P, Jilbert AR, Marmion BP. Correlation between liver histology and markers of hepatitis B virus replication in infected patients: a study by in situ hybridization. Hepatology. 1984;4(1):20-24.

18. Lau JY, Naoumov NV, Alexander GJ, Williams R. Rapid detection of hepatitis B virus DNA in liver tissue by in situ hybridisation and its combination with immunohistochemistry for simultaneous detection of HBV antigens. J Clin Pathol. 1991;44(11):905-908.

19. Loriot MA, et al. Persistence of hepatitis B virus DNA in serum and liver from patients with chronic hepatitis B after loss of HBsAg. J Hepatol. 1997;27(2):251-258.

20. Yeh CT, Chiu HT, Chu CM, Liaw YF. G1 phase dependent nuclear localization of relaxed-circular hepatitis B virus DNA and aphidicolin-induced accumulation of covalently closed circular DNA. JMed Virol. 1998;55(1):42-50.

21. Nuriya H, et al. Detection of hepatitis B and C viruses in almost all hepatocytes by modified PCR-based in situ hybridization. J Clin Microbiol. 2010;48(11):3843-3851.

22. O'Leary JJ, et al. PCR in situ hybridisation detec- 
tion of HPV 16 in fixed CaSki and fixed SiHa cell lines. JClin Pathol. 1994;47(10):933-938.

23. Zhong Y, et al. A novel method for detection of HBVcccDNA in hepatocytes using rolling circle amplification combined with in situ PCR. BMC Infect Dis. 2014;14(1):608.

24. Player AN, Shen LP, Kenny D, Antao VP, Kolberg JA. Single-copy gene detection using branched DNA (bDNA) in situ hybridization. J Histochem Cytochem. 2001;49(5):603-611.

25. Kenny D, Shen LP, Kolberg JA. Detection of viral infection and gene expression in clinical tissue specimens using branched DNA (bDNA) in situ hybridization. J Histochem Cytochem. 2002;50(9):1219-1227.

26. Newbold JE, et al. The covalently closed duplex form of the hepadnavirus genome exists in situ as a heterogeneous population of viral minichromosomes. J Virol. 1995;69(6):3350-3357.

27. Summers J, Smith PM, Horwich AL. Hepadnavirus envelope proteins regulate covalently closed circular DNA amplification. J Virol. 1990;64(6):2819-2824.

28. Lentz TB, Loeb DD. Roles of the envelope proteins in the amplification of covalently closed circular DNA and completion of synthesis of the plus-strand DNA in hepatitis B virus. J Virol. 2011;85(22):11916-11927.

29. Lau JY, Bain VG, Davies SE, Alexander GJ, Wil- liams R. Export of intracellular HBsAg in chronic hepatitis $B$ virus infection is related to viral replication. Hepatology. 1991;14(3):416-421.

30. Hadziyannis S, Gerber MA, Vissoulis C, Popper H. Cytoplasmic hepatitis B antigen in "groundglass" hepatocytes of carriers. Arch Pathol. 1973;96(5):327-330.

31. Gerken G, Manns M, Gerlich WH, Hess G, Meyer zum Buschenfelde KH. Immune blot analysis of viral surface proteins in serum and liver of patients with chronic hepatitis B virus infection. JMed Virol. 1989;29(4):261-265.

32. Hsu HC, et al. Biologic and prognostic significance of hepatocyte hepatitis B core antigen expressions in the natural course of chronic hepatitis B virus infection. J Hepatol. 1987;5(1):45-50.

33. Guidotti LG, Martinez V, Loh YT, Rogler $\mathrm{CE}$, Chisari FV. Hepatitis B virus nucleocapsid particles do not cross the hepatocyte nuclear membrane in transgenic mice. J Virol. 1994;68(9):5469-5475.

34. Yeh CT, Wong SW, Fung YK, Ou JH. Cell cycle regulation of nuclear localization of hepatitis $B$ virus core protein. Proc Natl Acad Sci U S A. 1993;90(14):6459-6463.

35. Chu CM, Yeh CT, Sheen IS, Liaw YF. Subcellular localization of hepatitis B core antigen in relation to hepatocyte regeneration in chronic hepatitis $\mathrm{B}$. Gastroenterology. 1995;109(6):1926-1932.
36. Trevisan A, Gudat F, Busachi C, Stocklin E, Bianchi L. An improved method for $\mathrm{HBcAg}$ demonstration in paraffin-embedded liver tissue. Liver. 1982;2(4):331-339.

37. Gowans EJ, Burrell CJ. Widespread presence of cytoplasmic HBcAg in hepatitis B infected liver detected by improved immunochemical methods. JClin Pathol. 1985;38(4):393-398.

38. Luckenbaugh L, Kitrinos KM, Delaney WE 4th, $\mathrm{Hu}$ J. Genome-free hepatitis B virion levels in patient sera as a potential marker to monitor response to antiviral therapy. J Viral Hepat. 2015;22(6):561-570.

39. Wang Y, et al. Different expression of hepatitis B surface antigen between hepatocellular carcinoma and its surrounding liver tissue, studied using a tissue microarray. J Pathol. 2002;197(5):610-616.

40. Tu T, et al. Clonal expansion of hepatocytes with a selective advantage occurs during all stages of chronic hepatitis B virus infection. J Viral Hepat. 2015;22(9):737-753.

41. Florell SR, et al. Preservation of RNA for functional genomic studies: a multidisciplinary tumor bank protocol. Mod Pathol. 2001;14(2):116-128.

42. Cai D, Nie H, Yan R, Guo JT, Block TM, Guo H. A southern blot assay for detection of hepatitis $B$ virus covalently closed circular DNA from cell cultures. Methods Mol Biol. 2013;1030:151-161. 